\title{
Improving the Trajectory Tracking Performance of Autonomous Orchard Vehicles Using Wheel Slip Compensation
}

\author{
${ }^{*}$ Gokhan Bayar ${ }^{\mathrm{a}, \mathrm{b}, \mathrm{c}}$, Marcel Bergerman ${ }^{\mathrm{b}}$, E. ilhan Konukseven ${ }^{\mathrm{a}}$, A. Bugra Koku ${ }^{\mathrm{a}}$ \\ gbayar@gmail.com, marcel@cmu.edu, konuk@metu.edu.tr, kbugra@metu.edu.tr \\ ${ }^{a}$ Mechanical Engineering Department, Middle East Technical University, Dumlupinar Bulvari, \\ Cankaya, 06800, Ankara, Turkey \\ ${ }^{\mathrm{b}}$ Field Robotics Center, Robotics Institute, Carnegie Mellon University, 5000 Forbes Avenue, 15213- \\ 3890 Pittsburgh, PA, USA \\ ${ }^{\mathrm{c}}$ Mechanical Engineering Department, Bulent Ecevit University, Incivez Mah., \\ 67100, Zonguldak, Turkey \\ *Corresponding author: Gokhan Bayar \\ Address: Mechanical Engineering Department, Bulent Ecevit University, Incivez Mah., \\ 67100, Zonguldak, Turkey. \\ Tel.: +(90)372-257-4010/1223 \\ e-mail: gbayar@gmail.com
}

\begin{abstract}
In this paper, the effects of wheel slip estimation and compensation of trajectory tracking for orchard applications were investigated. A slippage estimator was developed and adapted into a car-like robot model. Steering and velocity commands were generated using a model-based control approach. The whole system was implemented and tested on an autonomous orchard vehicle that has steerable front wheels and actuated rear wheels. A high accuracy positioning system was used to estimate the longitudinal and lateral slip velocities while the vehicle is moving. A laser scanning range finder was placed at the front centre of the vehicle, which was used to detect rows of trees in the orchard. Procedures were first tested in a non-flat but open space, which was covered with snow. Then it was tested on an experimental orchard where the surface was covered with heavy mud and the vehicle was expected to follow trajectories that span multiple rows in the orchard. The vehicle detected individual trees as well as rows of trees to track the centre of each row and manoeuvred from one row to the next. The experimental results showed that trajectory tracking performance of the vehicle was enhanced via integrating a slippage estimator into the system model. Furthermore, using the slippage estimation in the system model increased the accuracy, repeatability and performance of the control system.
\end{abstract}

\section{Keywords}

Autonomous orchard vehicle; Slippage estimation; Row following; Turning.

\section{Nomenclature}

RTK-GPS real time kinematic - Global Positioning System

WD wheel drive

ROS Robot Operating System

$\mathrm{X}, \mathrm{y} \quad$ coordinate system in the centre of the rear axle of the vehicle

$\mathrm{X}, \mathrm{Y} \quad$ vehicle's current position 


$\begin{array}{rll}1 & \theta & \text { vehicle orientation } \\ 2 & \omega & \text { vehicle angular velocity } \\ 3 & \phi & \text { steering angle } \\ 4 & v & \text { forward velocity } \\ 5 & \mathrm{~L} & \text { distance between front and rear axles } \\ 6 & \mathrm{~V}_{\mathrm{LF}} & \text { longitudinal slip velocity of the front wheels } \\ 7 & \mathrm{~V}_{\mathrm{LR}} & \text { longitudinal slip velocity of the rear wheels } \\ 8 & \mathrm{~V}_{\mathrm{SF}} & \text { lateral component of the front slip velocity } \\ 9 & \mathrm{~V}_{\mathrm{SR}} & \text { lateral component of the rear slip velocity } \\ 10 & \beta_{\mathrm{F}} & \text { slip angle of the front } \\ 11 & \beta_{\mathrm{R}} & \text { slip angle of the rear } \\ 12 & \mathrm{X}_{\mathrm{d}}, \mathrm{y}_{\mathrm{d}} & \text { desired position values } \\ 13 & \theta_{\mathrm{d}} & \text { desired orientation value } \\ 14 & v_{\mathrm{d}} & \text { desired forward velocity of the vehicle } \\ 15 & \mathrm{R} & \text { turning arc radius } \\ 16 & \mathrm{y}_{\mathrm{L}} & \text { distance between desired trajectory and vehicle } \\ 17 & \mathrm{X}_{\mathrm{e}} & \text { longitudinal error } \\ 18 & \mathrm{y}_{\mathrm{e}} & \text { lateral error } \\ 19 & \theta_{\mathrm{e}} & \text { orientation error } \\ 20 & \mathrm{C}(\mathrm{s}) & \text { profile of the desired trajectory } \\ 21 & \Pi_{\mathrm{obs}} & \text { observed variables } \\ 22 & \Pi_{\mathrm{mes}} & \text { measured values } \\ 23 & \mathrm{e} & \text { observation error } \\ 24 & \mathrm{k}_{\mathrm{x}}, \mathrm{k}_{\mathrm{y}}, \mathrm{k}_{\Omega} & \text { control variables } \\ 25 & \Omega & \text { time-varying function } \\ 26 & \lambda_{1}, \lambda_{2} & \text { positive time-varying functions } \\ 27 & \tau_{1}, \tau_{2} & \text { positive design parameters } \\ 28 & \delta_{1}, \delta_{2} & \text { inclination angles of the test area } \\ 29 & v_{\mathrm{c}} & \text { controller for the forward velocity } \\ 30 & \phi_{\mathrm{c}} & \text { controller for the steering angle } \\ 31 & \mathrm{wo} / \mathrm{S} & \text { without slip estimation } \\ 32 & \mathrm{w} / \mathrm{S} & \text { with slip estimation } \\ 33 & & \\ & & \end{array}$

\section{1. Introduction}

In this study, slip estimation to enhance real-time trajectory tracking performance of an autonomous field vehicle/robot was investigated. This enhancement is to improve accuracy and repeatability in trajectory tracking performance of an autonomous ground vehicle. To achieve this, a proper mathematical model is required for the vehicle that contains the necessary sensing hardware. Proper integration of sensor information into the system model is the key factor that determines the performance of a model-based controller. If performance expectation is moderate, ignoring surface effects with a flat terrain assumption yields a simplified approach. These assumptions may hold better for vehicle/robots operating indoors where surfaces are generally flat and not slippery. However, such models have restricted use in outdoors where the terrain is not necessarily smooth and slippage effect is more dramatic on the robot. In order to enhance trajectory tracking performance, by decreasing positioning errors and producing less abrupt control signals for steering, slippage information should be 48 taken into account. 
This paper presents a method for increasing the trajectory tracking performance for a vehicle used in orchards. The method proposed in this work includes the following steps: vehicle modelling, slippage estimation, stability check, and controller design for steering and velocity control. To improve tracking performance, a model-based controller is used where the estimated sideslip velocity is fed into the system model. The stability of the system is checked by using the Lyapunov approach. Experiments are conducted in both open space and in an experimental orchard-like environment using an autonomous vehicle. To observe the effects of slip estimation, these experiments are conducted with and without taking slippage estimation into account.

The paper is organised as follows: Section 2 presents the literature review followed by the statement of problem that is addressed in this study. Section 4 presents kinematic modelling and section 5 explains the details of estimation process of sideslip angles. Section 6 introduces controller design while the desired trajectory generation is given in Section 7. Prior to the concluding remarks, experimental results including both open space and orchard studies are presented.

\section{Literature Review}

In literature, trajectory tracking control with slip compensation is used to improve vehicles' performance under non-ideal and hence realistic conditions. Prior to the describing the proposed approach, a summary of research focusing on trajectory generation, tracking control, slippage definition and implementation for autonomous field robots is presented.

Lindgren et al., (2002), modelled an autonomous guided agricultural vehicle and considered slippage. Experiments were conducted in an open space using a four-wheeled mobile robot equipped with a vision system, odometer and torque sensors. Relationships between torque and slip were investigated where a traction model was also adopted into the system model. It was shown that navigation accuracy could be improved by integrating slippage information into the system model. The method of Lindgren et al., (2002) was further improved (Lenain et al., 2010) for navigation at higher speeds. Lenain et al., (2006) introduced an estimator for obtaining sideslip angles incorporating a high accuracy positioning system (RTK-GPS). A sideslip observer was integrated into this system model where tests were run using an autonomous farm tractor. During the experiments, the farm tractor was manually driven and the path was recorded by using the RTK-GPS at the end of which model parameters were then estimated.

Chen and Hsieh, (2008), proposed a sideslip estimation methodology based on an extended Kalman filter and a bicycle model. Sideslip angle was estimated for different driving test manoeuvres in a simulation environment. Baffet et al., (2008) estimated dynamic variables of a vehicle in order to enhance vehicle safety. The test platform was a modified commercial vehicle, which was equipped with various sensors such as accelerometer, gyroscopes, noncontact optical sensor, dynamometric hubs, incremental encoders and GPS. In addition to longitudinal and lateral tire forces, heading velocity and sideslip angles of the vehicle were used for real-time estimation of the cornering stiffness values. Grip et al., (2009) developed a sideslip observer for a four wheeled vehicle. A nonlinear sideslip observer was constructed by using dynamic and kinematic properties of the vehicle. Sideslip angle and friction parameters were estimated in real-time based on the proposed observer. Accelerations in lateral and longitudinal directions, angular velocity, steering angle and wheel speed information were used in the estimation process. However, the proposed method is not tested on a real robot. A 
trajectory tracking control method for use in agricultural tractors was introduced by Eaton et al., (2009). To improve the tracking performance and operational safety, a robust sliding mode control approach was developed where effects of slippage are taken into consideration. The procedure introduced was tested in simulation but was not tested using a tractor working in an agricultural application. Iijima et al., (2010) proposed a different perspective for estimating and integrating sideslip angles into dynamic vehicle model for improving traffic safety. Velocity, acceleration and yaw rate of the vehicle were used to estimate the sideslip without considering the steering information. In the modelling part of their study, a bicycle model was used. Experiments were conducted on a commercial vehicle on which CCD cameras, accelerometer and GPS were mounted. The experiments were performed on both dry and wet surfaces. Ryu et al., (2010), estimated vehicle sideslip angle by using an integration of GPS and INS systems. Their method was developed based on bicycle model. Experiments were conducted on a four-wheel commercial vehicle. Autonomous path tracking for the farm vehicles was studied by Matveev et al., (2010). It was shown that tracking a desired path is strongly affected by wheel slip. Path tracking task was achieved by using a sliding mode controller. However, the proposed method and control algorithm were only tested in a simulated environment, and not implemented on a real vehicle.

Concerning the specific area of autonomous orchard vehicles, Hamner et al., (2011) proposed a row detection system. Their system used a laser scanner range finder as the data source. Rows of trees were created using first order line representation. A more practical obstacle detection system which included tree and row detection and vehicle localisation approaches was proposed by Freitas et al., (2012). The system was tested using an autonomous orchard vehicle. A long distance autonomous trajectory tracking for a wheeled orchard vehicle was investigated by Bayar, (2013). A new perspective for developing turning geometry and desired steering angle representation was proposed and experimental results, including more than $15 \mathrm{~km}$ autonomous drives in an orchard environment, were presented. Barawid et al., (2007) developed an automatic guidance system for autonomous orchard applications. The system developed enabled the vehicle to navigate within its surrounding and travel between tree rows. In order to recognize trees and tree rows, a Hough transform based recognition system is introduced. Bayar et al., (2015) recently introduced a new method for detecting trees and rows of trees. Their study focused on controlling an autonomous orchard vehicle.

Thus, as seen from the literature, performance improvement of trajectory tracking for field vehicles/robots is an active field of research. How our work presented here differs from previous research is that the effects of slippage on trajectory tracking task in orchard environment are investigated under challenging real world conditions. These effects were observed on different types of surface and performance change in trajectory tracking due to slippage effects was studied.

\section{Problem Statement}

Autonomous self-driven orchard vehicles (i.e. a field robot tuned to operate in orchards) are being integrated into modern fruit production operations. The concept is that they can be used in fully automated operations such as mowing and spraying, etc. without an operator in sight. Moreover, these platforms may assist workers in routine operations such as pruning, thinning, tree maintenance, and harvesting. All these applications are based on the vehicle driving up along one row, turning at the end of the row, and entering the next row. The challenges are:

- Safely detecting trees and rows of trees. 
- Detecting tree branches, tall grass, and missing trees.

- Localising the vehicle inside the current row; following the desired trajectory that lies inside the consecutive rows; reliably creating a desired trajectory for manoeuvring from one row to another.

- Achieving all these operations in slippery, muddy, or snowy conditions on uneven surfaces.

Thus, one of the goals of this study was to detect trees and rows of trees and to control a wheeled vehicle to track a desired trajectory in an orchard environment. A typical orchard view is shown in Fig. 1-a. The desired trajectory which lies in the middle of two neighbour tree rows is depicted in Fig. 1-b.

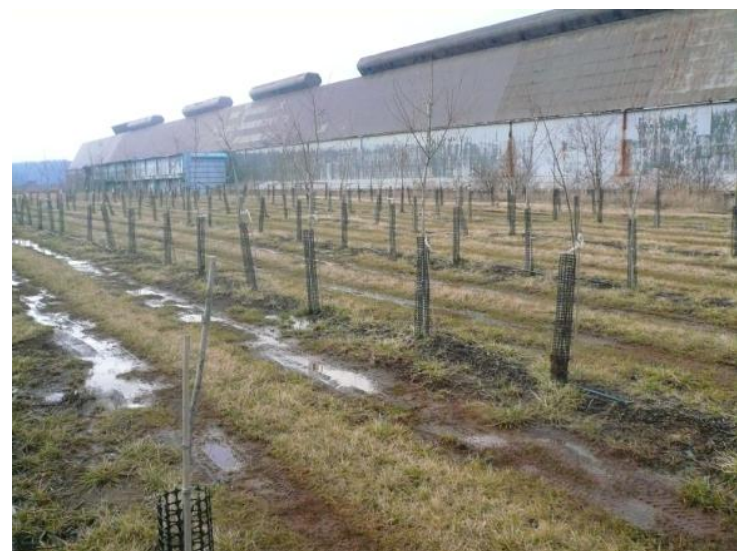

(a)

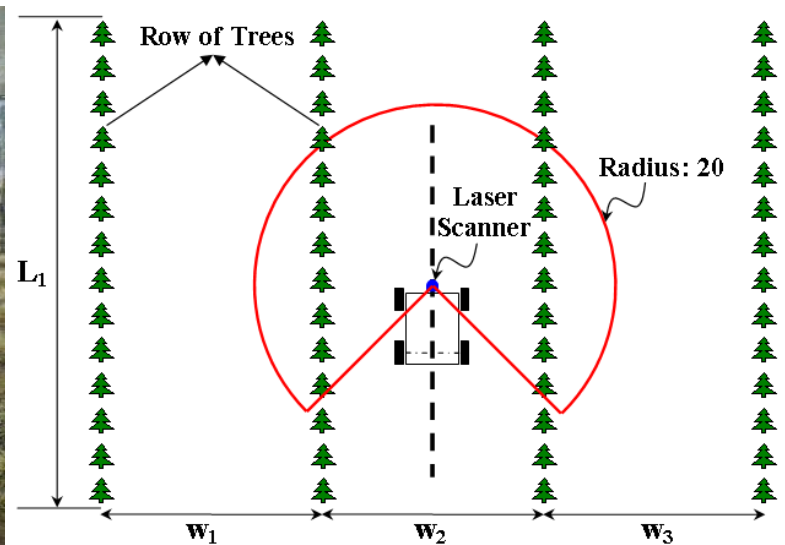

(b)

Fig. 1. (a) General view of the orchard with slippery surface, (b) Desired trajectory of the autonomous vehicle inside the orchard. Row lengths and widths are indicated by $\mathrm{L}_{1}$ and $\mathrm{w}_{\mathrm{i}}$, respectively. Radius shows the scanning range of laser scanning range finder.

The autonomous vehicle used in this study is shown in Fig. 2 (a). The 2WD vehicle had steerable wheels on the front and rear wheels were actuated by a single 48 -volt dc electric motor. Eight, 6-volt batteries that can supply a maximum of $75 \mathrm{~A}$ current are located in the vehicle. The weight, width, length, height and wheel base of the vehicle are $545 \mathrm{~kg}, 1500 \mathrm{~mm}$, $3040 \mathrm{~mm}, 1270 \mathrm{~mm}$ and $2057 \mathrm{~mm}$, respectively. The vehicle was equipped with a high accuracy positioning system (Applanix POS-LV RTK-GPS, manufactured by Applanix Company located in Richmond Hill, ON, Canada) providing position information to $10 \mathrm{~mm}$ resolution. RTK-GPS was used for the data source of the slippage estimation and as the ground truth. Encoders were attached to both the steering system and rear wheels. A laser range finder (Sick LMS111, manufactured by Sick Company located in Minneapolis, MN, USA) is installed in front of the vehicle to detect obstacles and trees. The scanning angle, scanning frequency and angular resolution of the laser range finder were $270^{\circ}, 25 \mathrm{~Hz}$ and $0.25^{0}$, respectively. The operating range of the laser scanner was $0.5 \mathrm{~m}$ to $20 \mathrm{~m}$. In order to obtain terrain inclination in two directions an inclination sensor (CHR-6dm, manufactured by Pololu Company located in Las Vegas, NV, USA) was used. All the communication and computational routines were implemented in ROS (Robot Operating System) (ROS, 2015) running on a single board Linux box. Note that ROS provides libraries, device drivers, packages and so many open source tools to give opportunity to the researchers and software developers create robot applications. 


\section{Kinematic Modelling}

2

The kinematic model of the vehicle (Fig. 2-b) can be given in the following form:

$$
\begin{aligned}
& \dot{\mathrm{x}}=v \cos \theta \\
& \dot{\mathrm{y}}=v \sin \theta \\
& \dot{\theta}=\omega=\frac{v}{\mathrm{~L}} \tan \phi
\end{aligned}
$$

where $\mathrm{x}$ and $\mathrm{y}$ are the Cartesian coordinates of the centre of the rear axle of the vehicle, $\theta$ is the vehicle orientation with respect to the fixed frame $(\mathrm{X}, \mathrm{Y}), \omega$ is the vehicle angular velocity, $\phi$ is the steering angle, and $v$ is the forward velocity. Distance between front and rear axles is indicated by L. Front axle coordinates are presented by $\mathrm{x}_{\mathrm{f}}$ and $\mathrm{y}_{\mathrm{f}}$.

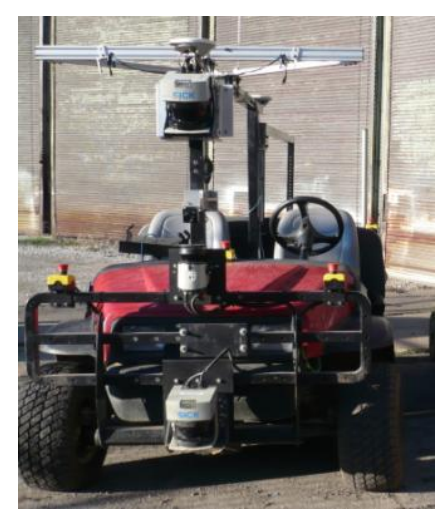

(a)

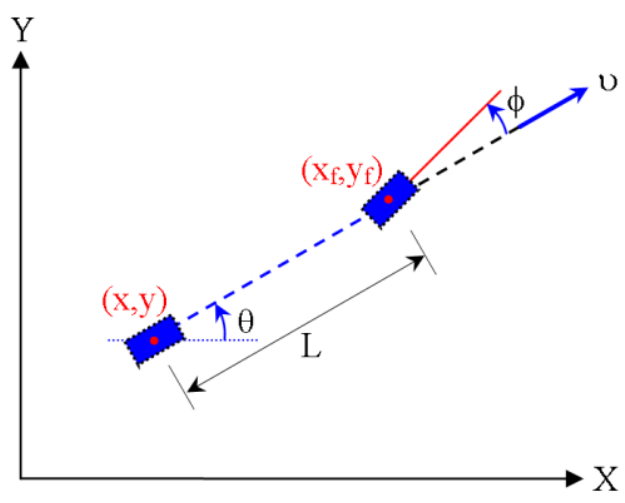

(b)

Fig. 2. (a) Four wheeled orchard vehicle used in this study, (b) kinematic model of the vehicle.

Slippage can be included in the kinematic model given in Eq. (1) by introducing longitudinal and lateral slip velocities. Longitudinal slip velocities of the front and rear wheels are indicated by $\mathrm{V}_{\mathrm{LF}}$ and $\mathrm{V}_{\mathrm{LR}}$, respectively (Fig. 3). Lateral components of the slip velocities are shown by $\mathrm{V}_{\mathrm{SF}}$ and $\mathrm{V}_{\mathrm{SR}}$, respectively. Slip angles of the front and rear axles are described by $\beta_{\mathrm{F}}$ and $\beta_{R}$, respectively. In Fig. 3, the desired position and orientation values are defined by $\left(x_{d}\right.$, $\mathrm{y}_{\mathrm{d}}$ ) and $\theta_{\mathrm{d}}$, respectively. The centre of turning arc is shown by point $\mathrm{A}$ and $\mathrm{R}$ is the turning arc radius. The distance between desired trajectory and vehicle is defined by $\mathrm{y}_{\mathrm{L}}$. Note that the objective of the control algorithm is to minimise this distance along a planned task. In this configuration, it is considered that the steering angle has positive value.

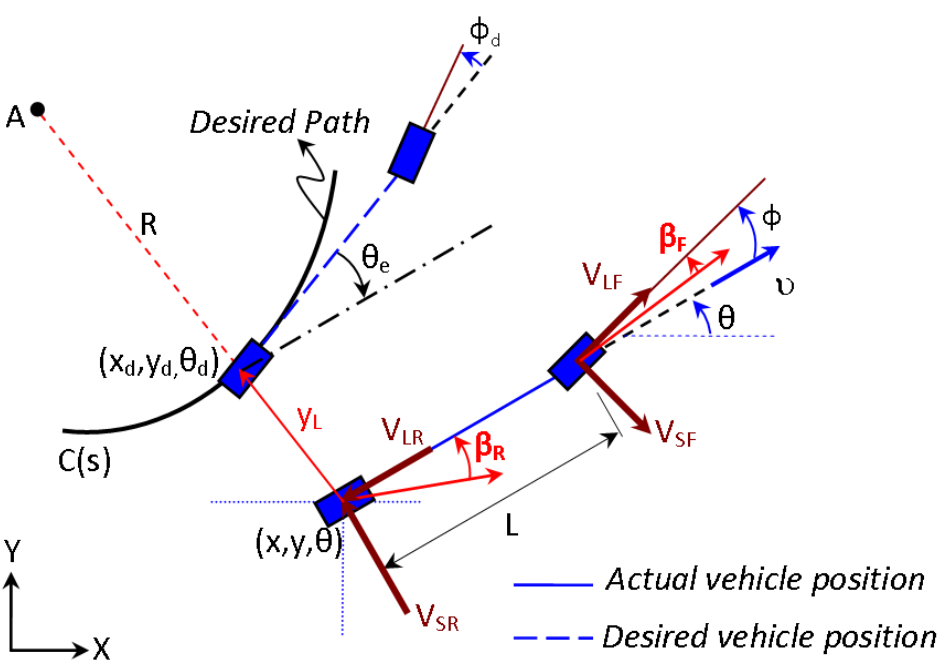

Fig. 3. Representation of the slippage and position components. 
Kinematic model of the vehicle with slip velocities can be defined as follows (Fang, 2004), (Fang et al., 2006), (Eaton et al., 2009), (Lenain et al., 2010):

$$
\begin{aligned}
& \dot{\mathrm{x}}=v \cos \theta-\mathrm{V}_{\mathrm{LR}} \cos \theta-\mathrm{V}_{\mathrm{SR}} \sin \theta \\
& \dot{\mathrm{y}}=v \sin \theta-\mathrm{V}_{\mathrm{LR}} \sin \theta+\mathrm{V}_{\mathrm{SR}} \cos \theta \\
& \dot{\theta}=\frac{v-\mathrm{V}_{\mathrm{LR}}}{\mathrm{L}}\left[\tan \beta_{\mathrm{R}}+\tan \left(\phi-\beta_{\mathrm{F}}\right)\right]
\end{aligned}
$$

4

Assuming that the vehicle is controlled to follow the desired trajectory defined by $\mathrm{q}=\left[\mathrm{x}_{\mathrm{d}}, \mathrm{y}_{\mathrm{d}}, \theta_{\mathrm{d}}\right]$. Errors occurring in longitudinal $\left(\mathrm{x}_{\mathrm{e}}\right)$ and lateral $\left(\mathrm{y}_{\mathrm{e}}\right)$ directions and orientation error $\left(\theta_{\mathrm{e}}\right)$ (Fig. 3) can be defined (Fang, 2004), (Fang et al., 2006), (Eaton et al., 2009), (Lenain et al., 2010) by:

$$
\left[\begin{array}{l}
x_{e} \\
y_{e} \\
\theta_{e}
\end{array}\right]=\left[\begin{array}{ccc}
\cos \theta & \sin \theta & 0 \\
-\sin \theta & \cos \theta & 0 \\
0 & 0 & 1
\end{array}\right]\left[\begin{array}{l}
x_{d}-x \\
y_{d}-y \\
\theta_{d}-\theta
\end{array}\right]
$$

The changes in errors in time in $\mathrm{x}, \mathrm{y}$ and $\theta$ are called the error dynamics of the system and can be presented via $\left(\dot{\mathrm{x}}_{\mathrm{e}}, \dot{\mathrm{y}}_{\mathrm{e}}, \dot{\theta}_{\mathrm{e}}\right)$, respectively. They can be obtained by differentiating the error components given in Eq. (3).

$$
\begin{aligned}
& \dot{\mathrm{x}}_{\mathrm{e}}=-\mathrm{v}+\omega \mathrm{y}_{\mathrm{e}}+\mathrm{v}_{\mathrm{d}} \cos \theta_{\mathrm{e}}+\varepsilon_{1} \\
& \dot{\mathrm{y}}_{\mathrm{e}}=\mathrm{v}_{\mathrm{d}} \sin \theta_{\mathrm{e}}-\omega \mathrm{x}_{\mathrm{e}}+\varepsilon_{2} \\
& \dot{\theta}_{\mathrm{e}}=v_{\mathrm{d}} \mathrm{C}(\mathrm{s})-\frac{\mathrm{v}}{\mathrm{L}} \tan \left(\phi-\beta_{\mathrm{F}}\right)+\varepsilon_{3}
\end{aligned}
$$

The components of the error dynamics $\left(\dot{\mathrm{x}}_{\mathrm{e}}, \dot{\mathrm{y}}_{\mathrm{e}}, \dot{\theta}_{\mathrm{e}}\right)$ are presented in terms of $\left(\varepsilon_{1}, \varepsilon_{2}, \varepsilon_{3}\right)$ in Eq. (4) for clarity. In this equation, $\varepsilon_{1}=\mathrm{V}_{\mathrm{LR}}+\mathrm{V}_{\mathrm{SR}} \sin 2 \theta, \quad \varepsilon_{2}=\mathrm{V}_{\mathrm{SR}}\left(1-2 \cos ^{2} \theta\right)$ and $\varepsilon_{3}=\frac{V_{L R}}{L} \tan \left(\phi-\beta_{F}\right)+\frac{V_{S R}}{L}$. The definition of $V_{S R}$ can be given as $V_{S R}=\left(v-V_{L R}\right) \tan \beta_{R} \cdot v_{d}$ is the desired forward velocity of the vehicle. During the experiments $v_{d}$ is chosen between 0.5 and $1.2 \mathrm{~m} \mathrm{~s}^{-1} . \mathrm{C}(\mathrm{s})$ is the profile of the desired trajectory. Two different desired trajectory profiles are presented in Fig. 6. Once the kinematic model with slip velocities is defined, the next step in the process is to estimate the sideslip angles.

\section{Sideslip Angle Estimation}

The estimation process aims to predict the front and rear sideslip angles indicated by $\beta_{\mathrm{F}}$ and $\beta_{\mathrm{R}}$ in Fig. 3. Sideslip angle estimation, which was proposed by Lenain et al. (2010), forms the basis of our work. A state observer is employed to estimate the slippage parameters in the model by using the information coming from RTK-GPS.

The state observer provides estimates of the two sideslip variables $\left(\mathrm{V}_{\mathrm{LR}}\right.$ and $\left.\mathrm{V}_{\mathrm{SR}}\right)$. Two observed variables are associated with the model given by $\Pi_{\mathrm{obs}}=(\mathrm{x}, \mathrm{y})_{\mathrm{obs}}$. They are obtained 
using the mathematical model presented here. The measured values of the observed variables are defined by $\Pi_{\text {mes }}=(\mathrm{x}, \mathrm{y})_{\text {mes }}$. The measured values are obtained by using the information coming from RTK-GPS. It is assumed that as long as RTK-GPS, steering and odometer systems supply adequate information; the following observation system can be used in the estimation procedure.

$$
\dot{\Pi}_{\mathrm{obs}}=\left[\begin{array}{c}
\dot{\mathrm{x}}_{\mathrm{obs}} \\
\dot{\mathrm{y}}_{\mathrm{obs}}
\end{array}\right]=\left[\begin{array}{l}
\left(\mathrm{v}-\mathrm{V}_{\mathrm{LR}}\right) \cos \theta-\mathrm{V}_{\mathrm{SR}} \sin \theta \\
\left(\mathrm{v}-\mathrm{V}_{\mathrm{LR}}\right) \sin \theta+\mathrm{V}_{\mathrm{SR}} \cos \theta
\end{array}\right]
$$

Here, $V_{L R}$ and $V_{S R}$ are the observed values of longitudinal and lateral slip velocities at the rear axel of the vehicle. Eq. (5) can be rearranged as follows:

$$
\dot{\Pi}_{\mathrm{obs}}=\left[\begin{array}{c}
u \cos \theta \\
v \sin \theta
\end{array}\right]+\left[\begin{array}{cc}
-\cos \theta & -\sin \theta \\
-\sin \theta & \cos \theta
\end{array}\right]\left[\begin{array}{c}
\mathrm{V}_{\mathrm{LR}} \\
\mathrm{V}_{\mathrm{SR}}
\end{array}\right] \Rightarrow \mathrm{P}=\mathrm{A}+(\mathrm{B})(\mathrm{u})
$$

Observation of slip velocities indicated by (u) in Eq. (6) can be defined in the following form:

$$
\mathrm{u}=\left[\begin{array}{c}
\mathrm{V}_{\mathrm{LR}} \\
\mathrm{V}_{\mathrm{SR}}
\end{array}\right]=\mathrm{B}^{-1}\left((\mathrm{G})(\mathrm{e})+\dot{\Pi}_{\mathrm{mes}}+\mathrm{P}-\mathrm{A}\right)
$$

where (e) represents the observation error defined by $\mathrm{e}=\Pi_{\mathrm{obs}}-\Pi_{\text {mes }}$. Error dynamics of the estimation process is specified by $\dot{\mathrm{e}}=(\mathrm{G})(\mathrm{e})$ (Lenain et al., 2010). (G) is a (2×2) matrix and is considered as a Hurwitz matrix that determines the observer gain regulating the settling time. Matrix $(\mathrm{G})$ is set up as a diagonal matrix so that the convergence of two variables observed can be decoupled.

Provided that $\mathrm{V}_{\mathrm{LR}}$ and $\mathrm{V}_{\mathrm{SR}}$ are obtained using Eq. (7), sideslip variables $\left(\beta_{\mathrm{F}}\right.$ and $\beta_{\mathrm{R}}$ ) can be calculated using Eqs. (2) and (4) as follows:

$$
\beta_{\mathrm{R}}=\tan ^{-1}\left(\frac{\mathrm{V}_{\mathrm{SR}}}{\mathrm{v}-\mathrm{V}_{\mathrm{LR}}}\right) \text { and } \beta_{\mathrm{F}}=\phi-\tan ^{-1}\left(\dot{\theta}\left(\frac{\mathrm{L}}{\mathrm{v}-\mathrm{V}_{\mathrm{LR}}}\right)-\tan \beta_{\mathrm{R}}\right)
$$

Orchard operations such as mowing, spraying, pruning and harvesting are usually performed with a forward vehicle velocity around $1.0 \mathrm{~m} \mathrm{~s}^{-1}$ (Davis 2012, Bayar et al., 2015). When the forward velocity of a four-wheeled vehicle (the rear and front wheels are used for driving and steering, respectively) is less than $1.0 \mathrm{~m} \mathrm{~s}^{-1}$, sideslip angle effects may be neglected (Joanny et al., 2003). The vehicle used in this study is driven via an electric motor instead of a combustion engine. The electric motor and battery packs were placed at the rear of the vehicle causing a lower the centre of gravity for the vehicle which provided less weight transfer. As a result wheel slip angle was affected by the less weight transfer and driving stability is improved. In this case, the sideslip angle of front (undriven) wheels can be neglected (Kanghyun et al., 2012), (Aleksander et al., 2000). Hence, the sideslip angle at the front of the vehicle is neglected $\left(\beta_{\mathrm{F}} \approx 0\right)$. 


\section{Control System Structure}

In this study, model-based control strategy was used. This control system generates required commands for driving and steering systems of the vehicle while the vehicle follows a reference trajectory.

The model-based control structure is based on Lyapunov stability approach (Fang, 2004, Fang et al., 2005, Fang et al., 2006, \& Eaton et al., 2009). Two Lyapunov functions are used. Firstly a Lyapunov function is defined as a function of time-varying errors in longitudinal and lateral directions:

$$
\mathrm{V}_{1}=\frac{1}{2} \mathrm{x}_{\mathrm{e}}^{2}+\frac{1}{2} \mathrm{y}_{\mathrm{e}}^{2}
$$

The derivative of this Lyapunov function is:

$$
\dot{\mathrm{V}}_{1}=\mathrm{x}_{\mathrm{e}} \dot{\mathrm{x}}_{\mathrm{e}}+\mathrm{y}_{\mathrm{e}} \dot{\mathrm{y}}_{\mathrm{e}}
$$

Substituting Eq. (4) into Eq. (10), the following relationship can be obtained:

$$
\dot{\mathrm{V}}_{1}=-\mathrm{k}_{\mathrm{x}} \mathrm{x}_{\mathrm{e}}^{2}-\mathrm{k}_{\mathrm{y}} \mathrm{y}_{\mathrm{e}}^{2}-\tau_{1} \mathrm{x}_{\mathrm{e}}{ }^{2} \lambda_{1}^{2}(\mathrm{t})-\tau_{2} \mathrm{y}_{\mathrm{e}}{ }^{2} \lambda_{2}^{2}(\mathrm{t})+\mathrm{x}_{\mathrm{e}} \varepsilon_{1}+\mathrm{y}_{\mathrm{e}}\left(\Omega+\varepsilon_{2}\right)
$$

where $\mathrm{k}_{\mathrm{x}}$ and $\mathrm{k}_{\mathrm{y}}$ are the control parameters, $\tau_{1}$ and $\tau_{2}$ are positive design parameters. The timevarying function $(\Omega)$ and the forward velocity of the vehicle $(v)$ are chosen as follows:

$$
\begin{aligned}
& \Omega=v_{d} \sin \theta_{e}+k_{y} y_{e}+\tau_{2} y_{e} \lambda_{2}^{2}(t) \\
& v=v_{d} \cos \theta_{e}+k_{x} x_{e}+\tau_{1} x_{e} \lambda_{1}^{2}(t)
\end{aligned}
$$

where $\lambda_{1}(\mathrm{t})$ and $\lambda_{2}(\mathrm{t})$ are positive time-varying functions and can be defined as $\lambda_{1}(\mathrm{t})>\left|\varepsilon_{1}\right|, \lambda_{2}(\mathrm{t})$ $>\left|\varepsilon_{2}\right|$. Equation (11) can also be written in the following form to check the Lyapunov stability criteria:

$$
\dot{\mathrm{V}}_{1} \leq-\mathrm{k}_{\mathrm{x}} \mathrm{x}_{\mathrm{e}}{ }^{2}-\mathrm{k}_{\mathrm{y}} \mathrm{y}_{\mathrm{e}}{ }^{2}-\tau_{1}\left(\left|\mathrm{x}_{\mathrm{e}}\right| \lambda_{1}(\mathrm{t})-\frac{1}{2 \tau_{1}}\right)^{2}-\tau_{2}\left(\left|\mathrm{y}_{\mathrm{e}}\right| \lambda_{2}(\mathrm{t})-\frac{1}{2 \tau_{2}}\right)^{2}+\frac{1}{4 \tau_{1}}+\frac{1}{4 \tau_{2}}+\mathrm{y}_{\mathrm{e}} \Omega
$$

The second Lyapunov function can be defined as follows:

$$
\mathrm{V}_{2}=\mathrm{V}_{1}+\frac{1}{2} \Omega^{2}
$$

The derivative of $\mathrm{V}_{2}$ and $\Omega$ can be derived as follows:

$$
\dot{\mathrm{V}}_{2}=\dot{\mathrm{V}}_{1}+\Omega \dot{\Omega}
$$

$$
\dot{\Omega}=v_{\mathrm{d}} \dot{\theta}_{\mathrm{e}} \cos \theta_{\mathrm{e}}+\dot{\mathrm{y}}_{\mathrm{e}}\left(\mathrm{k}_{\mathrm{y}}+\tau_{2} \lambda_{2}^{2}(\mathrm{t})\right)+2 \tau_{2} \mathrm{y}_{\mathrm{e}} \lambda_{2}(\mathrm{t}) \frac{\partial \lambda_{2}(\mathrm{t})}{\partial \mathrm{t}}
$$


Inserting Eq. (16) into Eq. (15) yields the following equality:

$$
\begin{aligned}
\dot{\mathrm{V}}_{2} \leq & -\mathrm{k}_{\mathrm{x}} \mathrm{x}_{\mathrm{e}}{ }^{2}-\mathrm{k}_{\mathrm{y}} \mathrm{y}_{\mathrm{e}}{ }^{2}-\tau_{1}\left(\left|\mathrm{x}_{\mathrm{e}}\right| \lambda_{1}(\mathrm{t})-\frac{1}{2 \tau_{1}}\right)^{2}-\tau_{2}\left(\left|\mathrm{y}_{\mathrm{e}}\right| \lambda_{2}(\mathrm{t})-\frac{1}{2 \tau_{2}}\right)^{2}+\frac{1}{4 \tau_{1}}+\frac{1}{4 \tau_{2}}+\mathrm{y}_{\mathrm{e}} \Omega+ \\
& \Omega\left(\mathrm{v}_{\mathrm{d}} \dot{\theta}_{\mathrm{e}} \cos \theta_{\mathrm{e}}+\dot{\mathrm{y}}_{\mathrm{e}}\left(\mathrm{k}_{\mathrm{y}}+\tau_{2} \lambda_{2}{ }^{2}(\mathrm{t})\right)+2 \tau_{2} \mathrm{y}_{\mathrm{e}} \lambda_{2}(\mathrm{t}) \frac{\partial \lambda_{2}(\mathrm{t})}{\partial \mathrm{t}}\right)
\end{aligned}
$$

In order to meet the Lyapunov stability criteria, it has to be shown that $\dot{\mathrm{V}}_{2} \leq 0$. This stability condition can be satisfied when the following equality is satisfied for the angular velocity of the vehicle.

$$
\omega=\frac{\mathrm{k}_{\Omega} \Omega+\mathrm{y}_{\mathrm{e}}+\mathrm{v}_{\mathrm{d}}{ }_{\mathrm{d}} \cos \theta_{\mathrm{e}} \mathrm{C}(\mathrm{s})+\left(\mathrm{k}_{\mathrm{y}}+\tau_{2} \lambda^{2}{ }_{2}(\mathrm{t})\right)\left(\mathrm{v}_{\mathrm{d}} \sin \theta_{\mathrm{e}}+\varepsilon_{2}\right)+2 \tau_{2} \mathrm{y}_{\mathrm{e}} \lambda_{2}(\mathrm{t}) \frac{\partial \lambda_{2}(\mathrm{t})}{\partial \mathrm{t}}}{\mathrm{v}_{\mathrm{d}} \cos \theta_{\mathrm{e}}+\mathrm{x}_{\mathrm{e}}\left(\mathrm{k}_{\mathrm{y}}+\tau_{2} \lambda^{2}{ }_{2}(\mathrm{t})\right)}
$$

where $\mathrm{k}_{\Omega}$ is the third control parameter. Lyapunov function now looks as follows:

$$
\dot{\mathrm{V}}_{2} \leq-\mathrm{k}_{\mathrm{x}} \mathrm{x}_{\mathrm{e}}^{2}-\mathrm{k}_{\mathrm{y}} \mathrm{y}_{\mathrm{e}}^{2}-\mathrm{k}_{\Omega} \Omega^{2}+\frac{1}{4 \tau_{1}}+\frac{1}{4 \tau_{2}}
$$

Finally, the controllers for the forward velocity $\left(v_{c}\right)$ and the steering angle $\left(\phi_{c}\right)$ of the vehicle, which depend on slip velocities $\left(\mathrm{V}_{\mathrm{LR}}, \mathrm{V}_{\mathrm{SR}}\right)$, are obtained from the following:

$$
\begin{aligned}
& v_{c}\left(V_{L R}, V_{S R}\right)=v_{d} \cos \theta_{e}+k_{x} x_{e}+\tau_{1} x_{e} \lambda_{1}^{2}(t) \\
& \phi_{c}\left(V_{L R}, V_{S R}\right)=\tan ^{-1}\left(\frac{L \omega}{v}\right)
\end{aligned}
$$

Three sets of controller parameters are used in this study. These parameters, which are presented in Table 1, are chosen according to the motion type. The first set of values is used in linear motion. The second and third sets are used during circular paths having small and large turning radiuses, respectively (see Figs. 5 and 6 for details).

Table 1. Control parameters.

\begin{tabular}{|c|c|c|c|}
\hline & $\mathrm{k}_{\mathrm{x}}$ & $\mathrm{k}_{\mathrm{y}}$ & $\mathrm{k}_{\Omega}$ \\
\hline Set-1 & 0.6 & 0.08 & 0.001 \\
\hline Set-2 & 0.12 & 0.12 & 0.02 \\
\hline Set-3 & 0.06 & 0.09 & 0.002 \\
\hline
\end{tabular}

The positive design parameters $\left(\tau_{1}\right.$ and $\left.\tau_{2}\right)$ are selected in agreement with the system behaviour.

The control structure is illustrated in Fig. 4. The information coming from the RTK-GPS is used to feed the slippage estimation and as the ground truth during both linear and curved motions. Linear motion is performed through the rows between the trees. During this motion the row detection system provides required information about the location of trees, rows of trees, position and orientation of the vehicle. While the vehicle performs a turning motion, the 
row detection system still works. However, it is not able to give any reliable information until the row detection system sees the trees and/or rows of trees. Therefore the position, orientation and slippage information during turning are obtained using encoders and RTKGPS, respectively. The vehicle localisation block shown in Figure 4 is fed by the data coming from the laser scanner, wheel and steering encoders, and position feedback block. The detail of this block is described in Bayar et al. (2015).

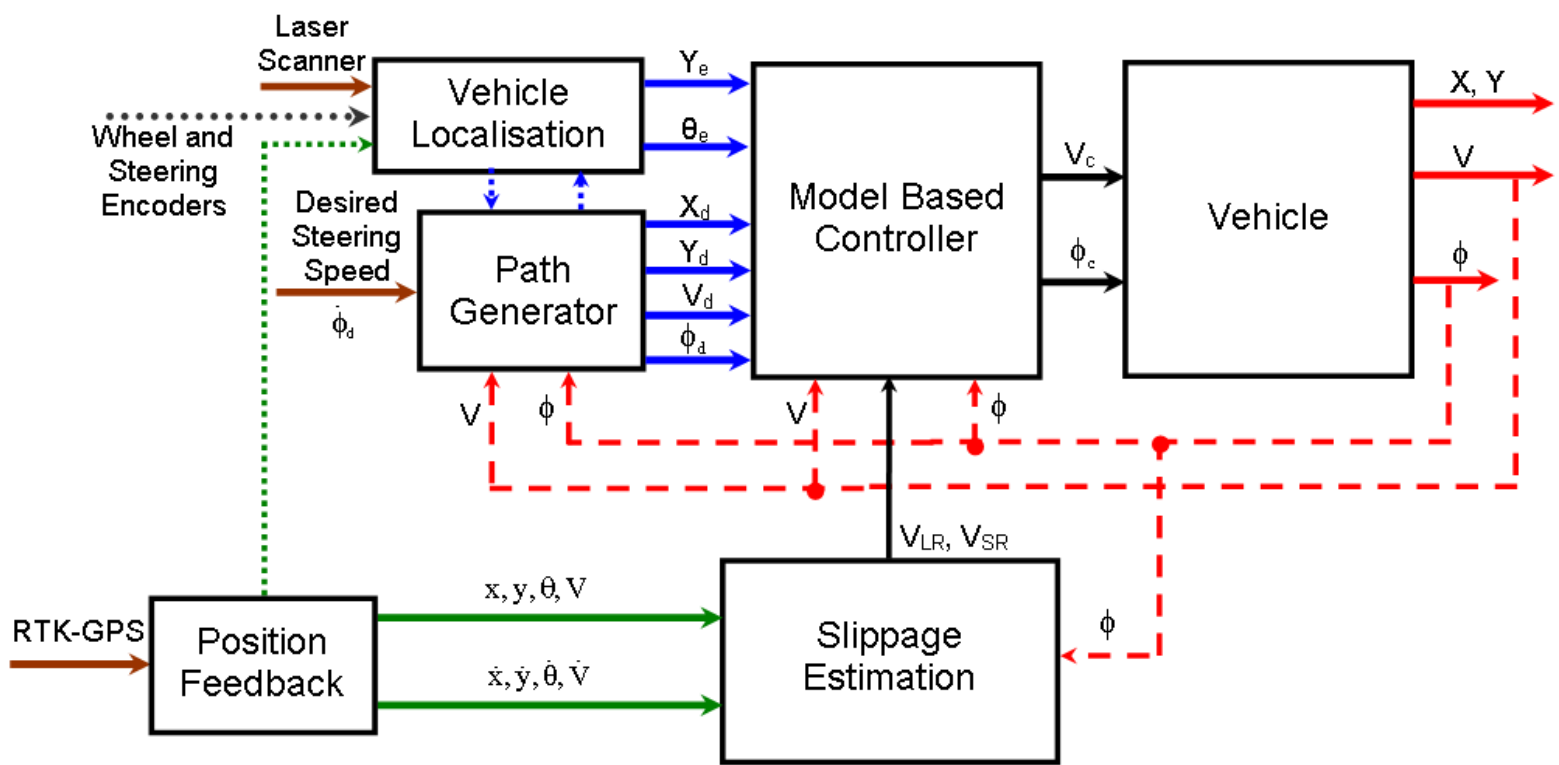

Fig. 4. Control system structure. Row detection system runs inside the vehicle localisation block.

\section{Desired Trajectory Generation for Turning}

When the row detection system observes that the vehicle is near the end point of a tree row it gets this information and the vehicle starts manoeuvring. The developed manoeuvre generator creates the desired trajectory and the controller creates the necessary turning control commands. Turning is continued until the point where the vehicle's heading becomes parallel to the next row to be entered.

While turning strategy was constructed, the followings were taken into account:

1 - Location of the vehicle while the vehicle performs manoeuvring.

2- Whether the vehicle is placed inside a predefined region or not (see Fig. 5) at the end of manoeuvring.

3- "Natural-looking" of the manoeuvring path.

Definition of "natural-looking" gives the necessary information about safe manoeuvring time, space and angle. If the manoeuvres of an experienced driver are recorded, a natural-looking path can be observed. The analysis of this recorded manoeuvre provides the required information for a complete turn between rows. Furthermore this analysis gives important other information related to the limits and the characteristics of the change of steering angle. Following these concerns, the turning trajectory generator develops a desired path according to the safety parameters, which are indicated in Fig. 5 by $S_{L}, S_{w}, S_{s}$ and $S_{F}$. These four parameters depend on the physical properties of the field and are determined prior to the operations in the field. 
1 Based on the results of many experiments it was concluded that the vehicle should locate 2 itself inside the predefined target region (as illustrated in Fig. 5). Once the vehicle was inside 3 the target region and had the desired heading angle, the row detection system was able to 4 detect the rows accurately.

Two similar turning geometries are used in this study as shown in Fig. 6. They are called as symmetrical (or bulb) turning (Fig. 6-a) and asymmetrical (or thumb) turning (Fig. 6-c). The symmetrical turning approach is easy to create and simple to adapt. It is a useful manoeuvring strategy in an open space however it may not be a good choose in an orchard due to its "unnatural-looking" behaviour. A "natural-looking" behaviour can be created using the thumb turning procedure. The procedure combines the simplicity of the bulb turn with usability in time and clothoid turning behaviour (Kanayama and Hartman, 1989, Shin and Singh, 1990). Such turning geometries are constructed using Dubins curves where two points on a plane is to be connected using the shortest curve (Dubins, 1957). In addition to desired trajectories, steering angle representations for the corresponding manoeuvring operations are illustrated in Fig. 6-b and 6-d. Ideal steering angles corresponding to the relating behaviour are given in the dashed lines (Figs. 6-b and 6-d). For practical reasons, sudden and high amplitude steering actions are not preferred. Such actions can be avoided by adapting a smoother steering profile. In this case a fourth order polynomial in the form of $\phi(t)=\mathrm{At}^{4}+\mathrm{Bt}^{3}+\mathrm{Ct}^{2}+\mathrm{Dt}+\mathrm{E}$ was used to generate smoother steering transitions. The parameters of the polynomial (i.e. A, B, C, D, E) are defined according to the characteristics of the steering system. 


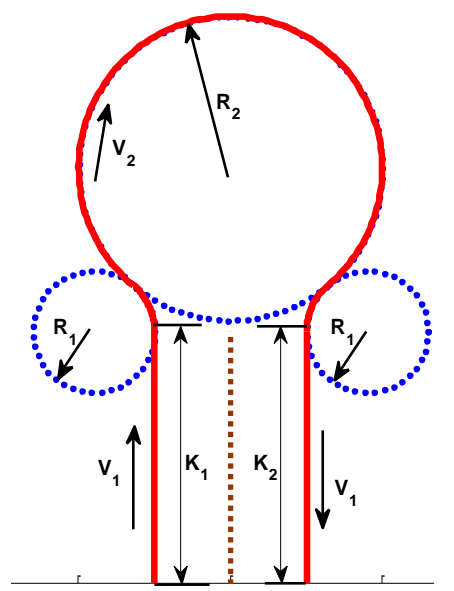

(a)

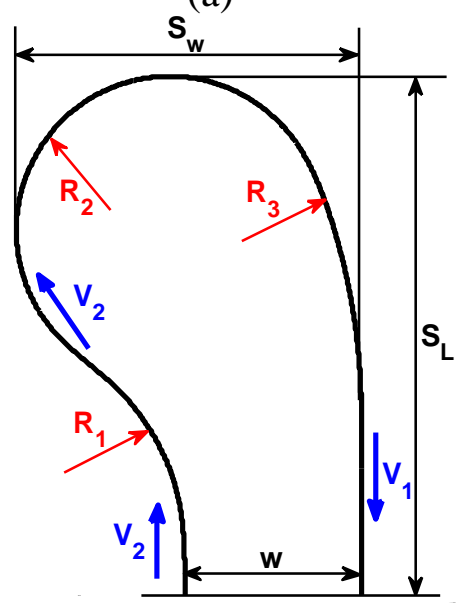

(c)

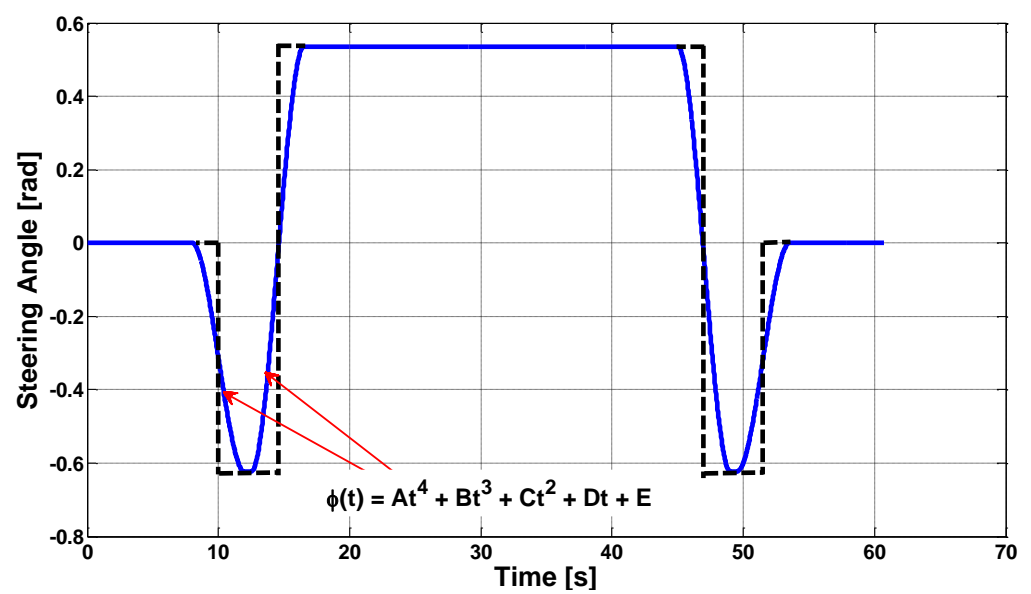

(b)

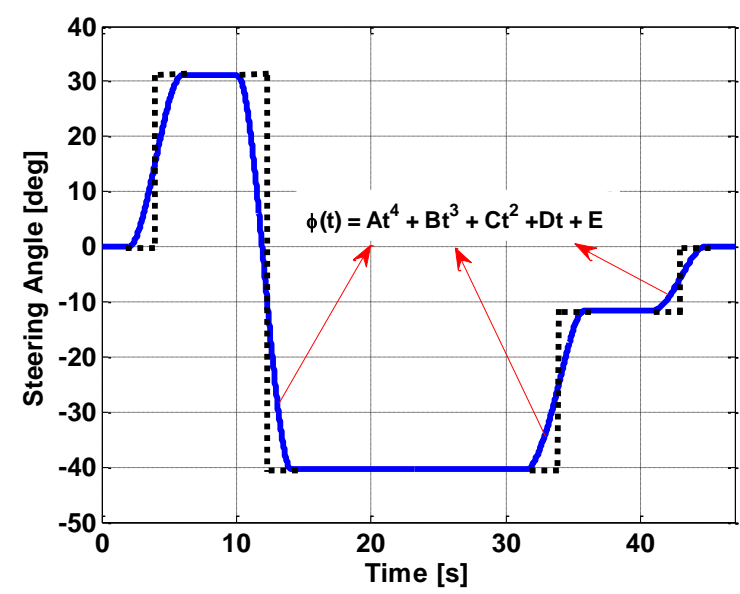

(d)

Fig. 6. (a) Bulb turn, (b) steering angle representation of bulb turn, (c) thumb turn, (d) steering angle representation of thumb turn.

\section{Experiments}

In order to investigate the effects of slippage on trajectory tracking performance, open space and orchard experiments were conducted during autumn and winter seasons. Open space experiments were conducted on snow-covered ground surface of the Schenley Park area at Carnegie Mellon University Campus, Pittsburgh, PA, USA. Orchard experiments were performed on the muddy surface of the experimental nursery located at the Robot City area in Pittsburgh.

\subsection{Open space experiments}

The open space experiment places were safe and suitable areas to test algorithms and conduct autonomous drive experiments since no trees were planted and no obstacles were placed in the area. In order to test the performance of the slippage estimation and the model-based control strategies, the vehicle was directed to follow the desired trajectory. In these experiments, the developed algorithm was responsible for determining the desired trajectories based on position feedback taken from RTK-GPS.

The experiments were conducted twice; once with and once without slippage estimation incorporated in the system model. The experimental area shown in Fig. 7 was totally covered 
with snow. Some parts of this area have inclined surfaces which can be represented by two
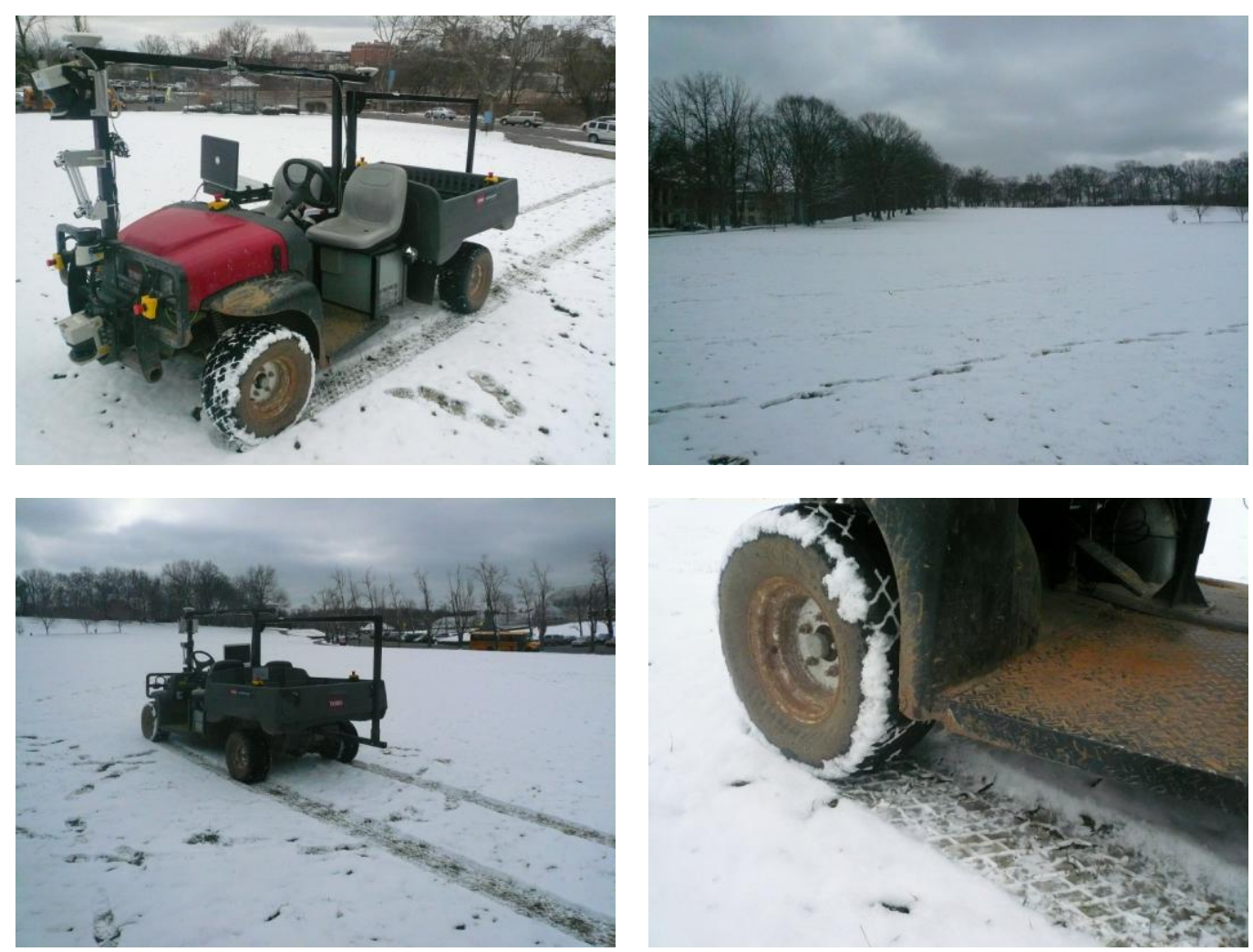

Fig. 7. Open space experiments conducted on the snow-covered ground surface. The images represent the general view of the experimental area which was covered with snow.

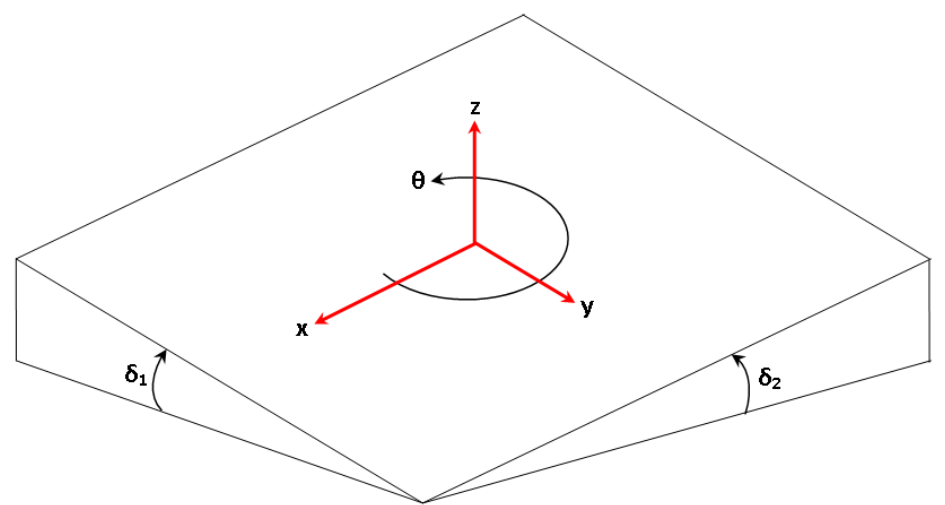

Fig. 8. Surface inclination angles $\left(\delta_{1}\right.$ and $\left.\delta_{2}\right)$ and the orientation of the vehicle $(\theta)$.

The experimental results are given in Fig. 9. They show the trajectories followed in two tests one with $(\mathrm{w} / \mathrm{S})$ and the other without (wo/S) slip estimation in the system model. The inclination angles measured while the vehicle travels through the actual trajectories are shown in Fig. 9(b). Desired and actual trajectories in longitudinal (x) and lateral (y) directions are given in Figs. 9(c) and 9(d), respectively. In these figures, curved and linear parts of the trajectories followed are also specified.

The longitudinal and lateral slip velocities estimated during the motion are shown in Fig. 10(a). Slip angles estimated at the rear of the vehicle are shown in Fig. 10(b). 


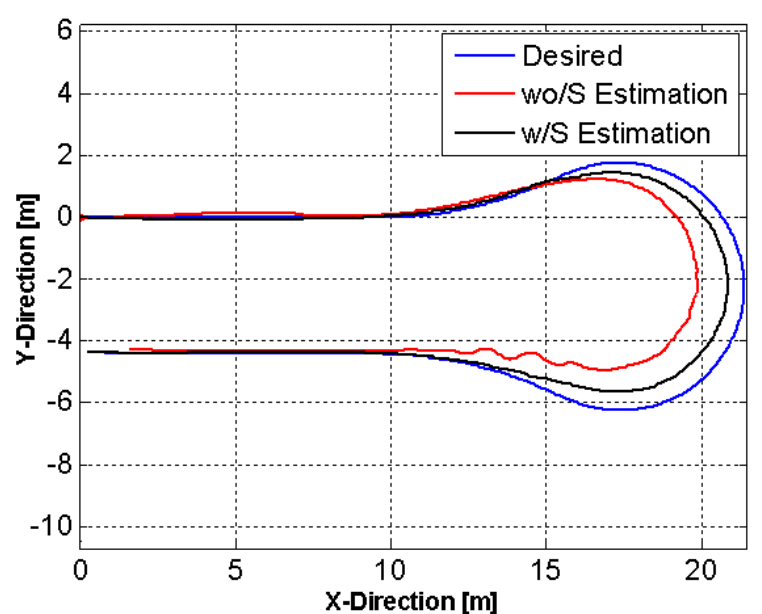

(a)

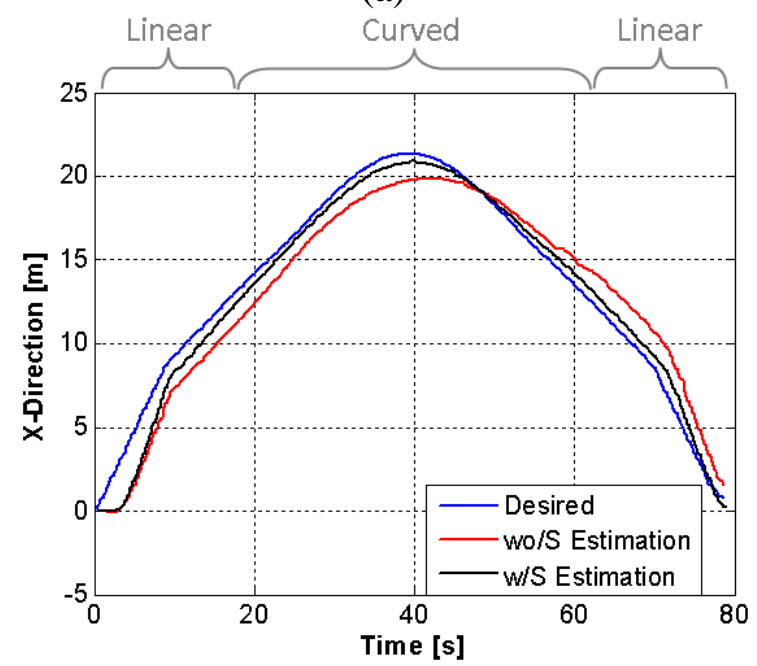

(c)

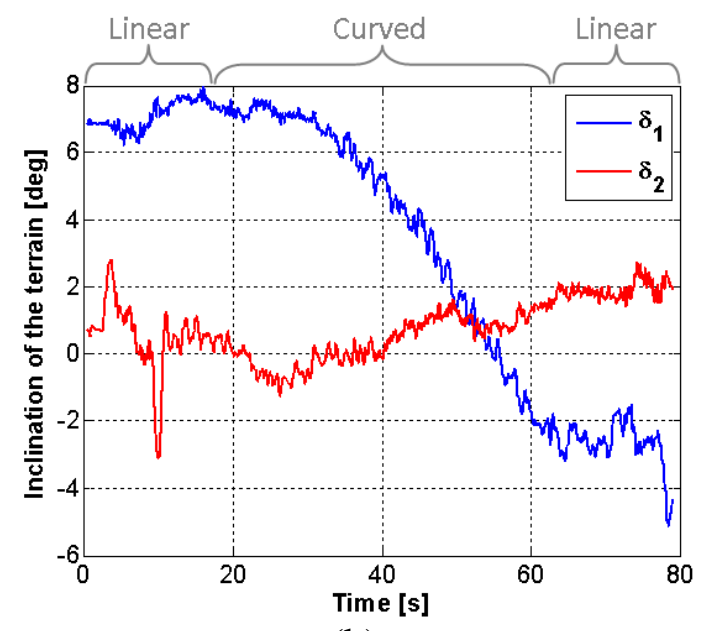

(b)

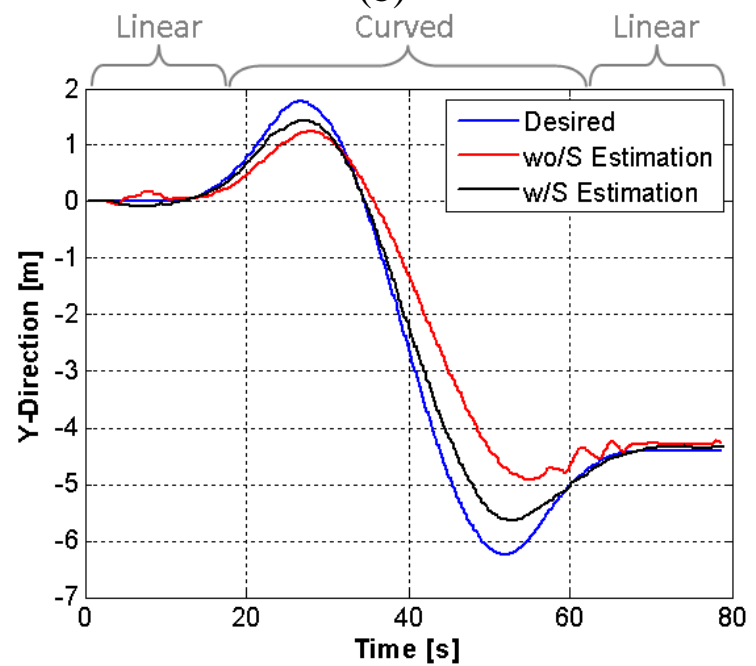

(d)

Fig. 9. Trajectory tracking experiment results performed in the snowy and inclined region. (a) Actual trajectories, (b) Inclination angles of the experimental region, (c-d) Actual trajectories in longitudinal (x) and lateral (y) directions. The notations "wo/S" and "w/S" specify without and with slip estimation.

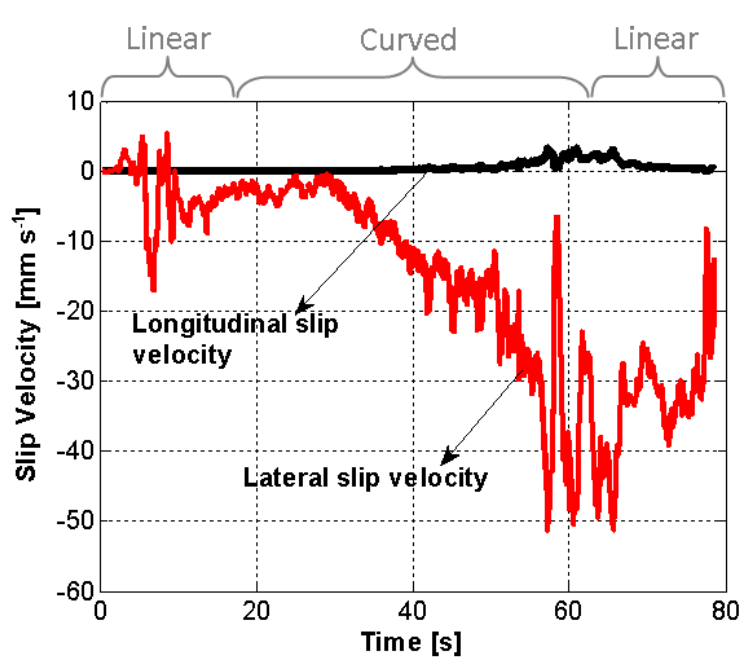

(a)

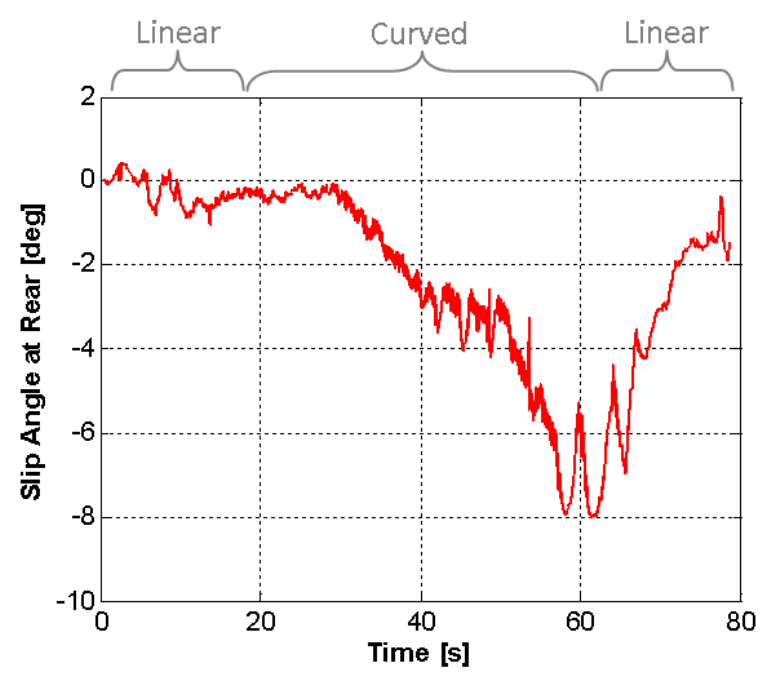

(b)

Fig. 10. (a) Longitudinal and lateral slip velocities estimated at the rear of the vehicle, (b) Slip angle estimated at the rear of the vehicle. 
While the vehicle tracks the desired trajectory in autonomous mode, the controller generated steering angle and forward velocity commands. These control commands recorded during the experiments are illustrated in Fig. 11. As seen in Fig. 11(a), when slippage was not taken into account, the error accumulated and the steering system carried out sharp steering actions to enable it to track the desired trajectory. However, if the slippage estimation procedure was used, the steering system controller produced smooth steering actions due to only small tracking errors.

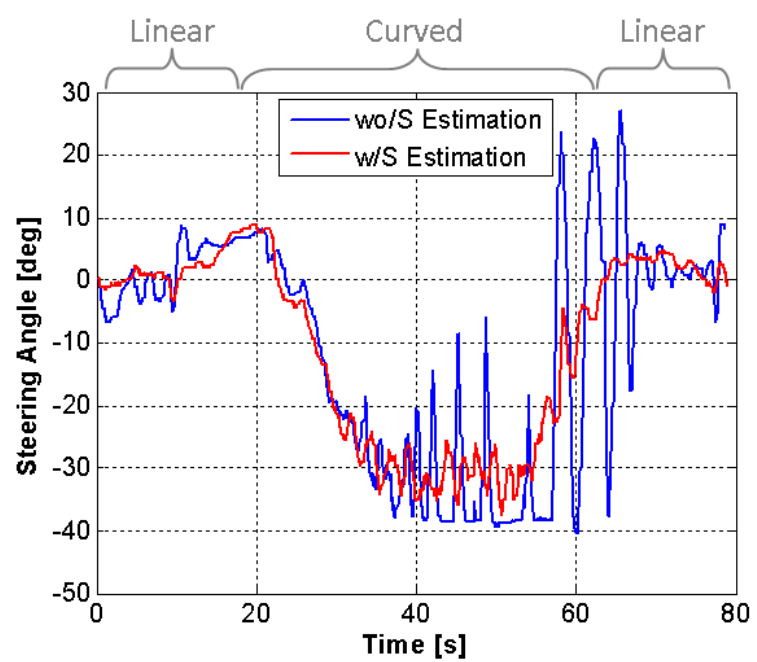

(a)

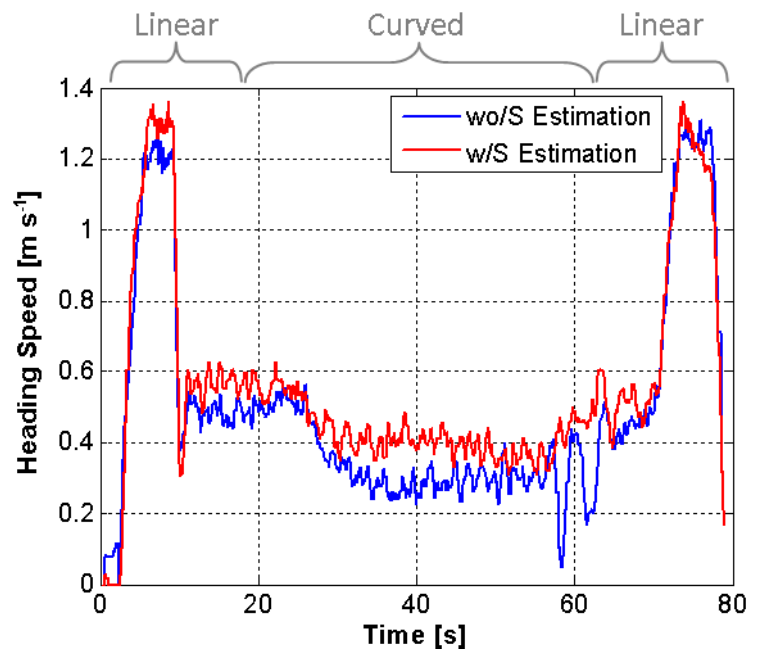

(b)

Fig. 11. (a) Steering angle, (b) heading velocity of the vehicle. The notations "wo/S" and "w/S" specify without and with slip estimation.

In addition to single turn performed in open space, multi-turn experiments were performed for a longer path in order to show the performance of the proposed method. In these experiments, the trajectory tracking performance of the vehicle was tested using the system model that includes the estimated slippage information. Desired and actual trajectories obtained in these experiments are shown in Fig. 12(a). The measured inclination angles of the test area are shown by $\delta_{1}$ and $\delta_{2}$ in Fig. 12(b). Desired and actual trajectories in longitudinal and lateral directions are shown in Fig. 13, respectively.

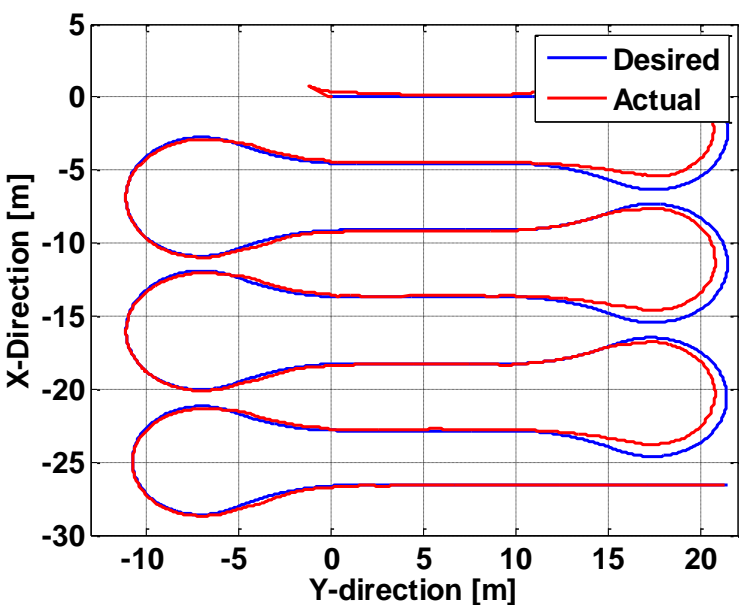

(a)

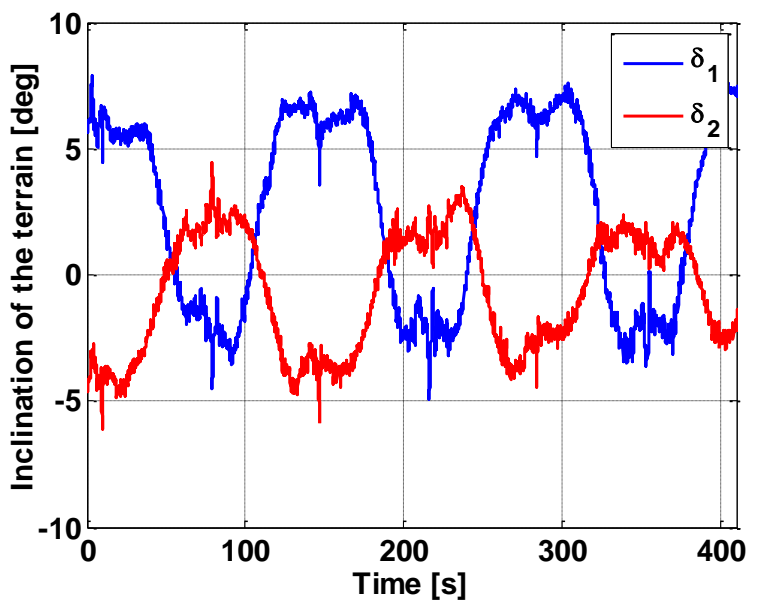

(b)

Fig. 12. Multi-turn experimental results obtained in the snowy region having inclination. In this experiment the slippage estimation algorithm is accompanied by the system model. (a) Trajectory tracking results, (b) measured inclination angles of the test area. 

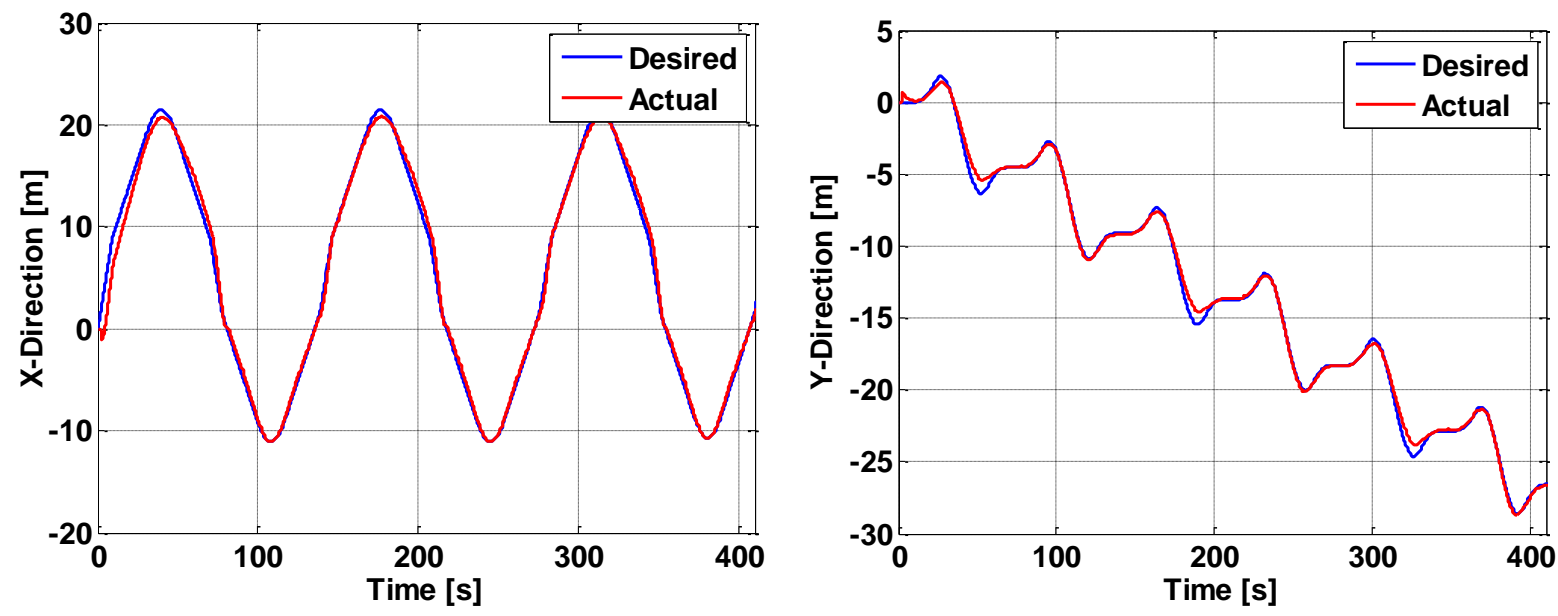

Fig. 13. Desired (Blue) and actual (Red) trajectories in $\mathrm{X}$ and $\mathrm{Y}$ directions.

Estimated longitudinal and lateral slip velocities, and sideslip angles are presented in Figs 14(a) and 14(b), respectively. Steering angle and forward velocity results are illustrated in Fig. 15.

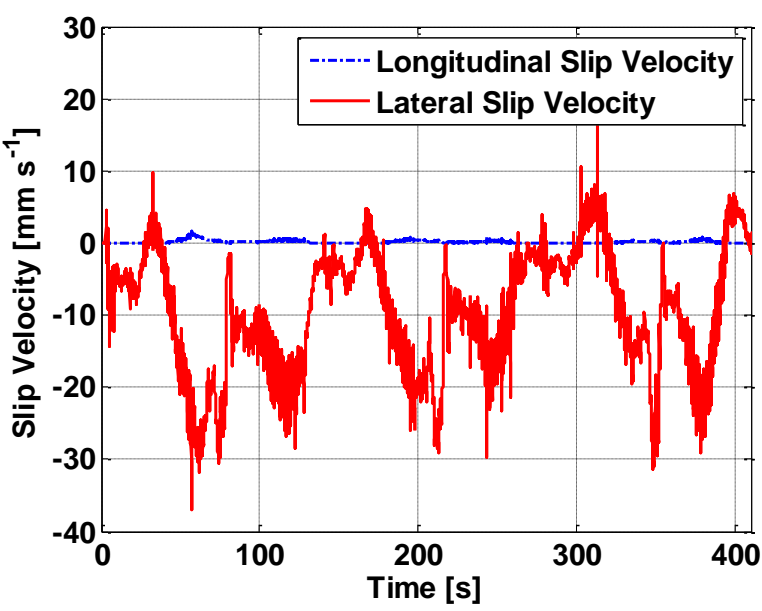

(a)

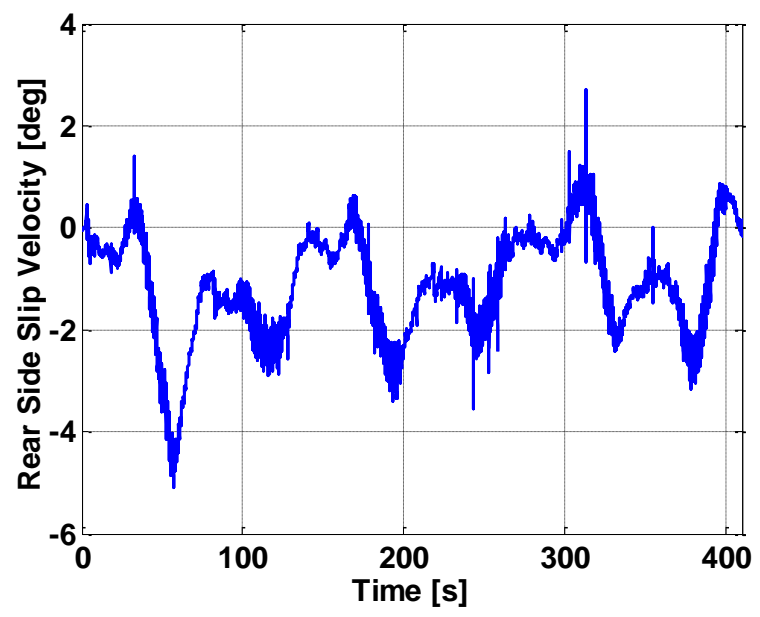

(b)

Fig. 14. (a) Estimated slip velocities in longitudinal and lateral directions, (b) estimated sideslip angles.

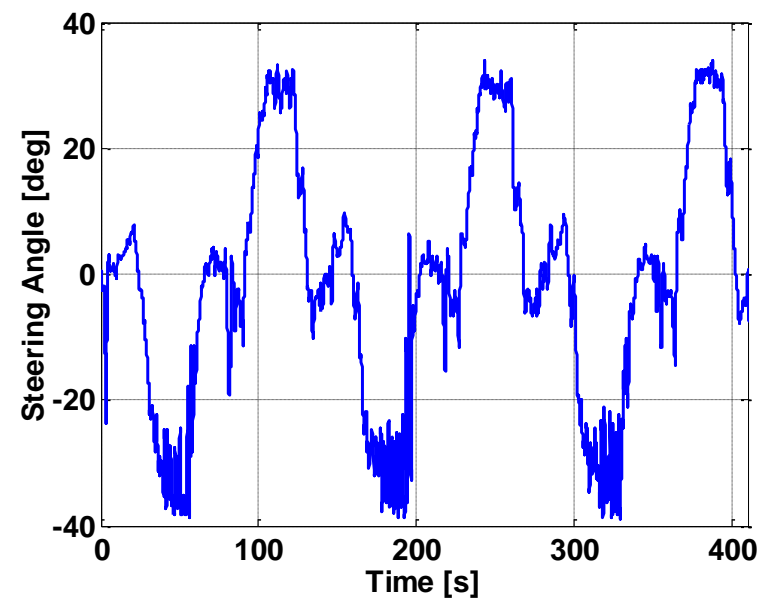

(a)

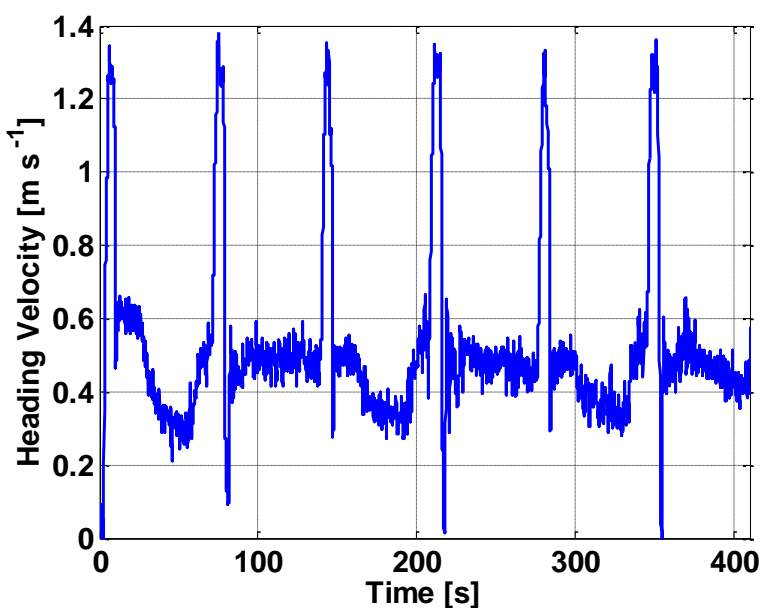

(b)

Fig. 15. (a) Steering angle, (b) heading velocity. 
The results indicated that incorporating the slippage information into the system model result in generation of smoother steering angle commands whereas heading velocity commands do not change noticeably. The system model proposed provided better trajectory tracking performance. The open space experiments used long trajectories with many turns. Despite some overshoots in the turns, which are acceptable for most practical purposes, no error accumulation was observed. It can be stated that the proposed method is suitable for adaptation in an autonomous vehicle designed for orchard applications.

\subsection{Orchard Experiments}

Orchard experiments were conducted in an orchard-style nursery which is a 72 ha site alongside the Monongahela River in Hazelwood, PA, USA. Figure 1(a) illustrates the experimental area.

In this part of the study, the objective was that the orchard vehicle should be able to follow desired trajectory between rows of trees with a high-level of consistent performance. To accomplish this, the system introduced in the previous sections of this paper was combined with the row detection system. As stated earlier, the row detection system was developed to detect trees and rows of trees and used a laser scanning range finder located in the mid-front of the vehicle. The developed system was capable of detecting tree trunks and creates lines that represent rows of trees together with their starting and finishing positions. This feature gave the vehicle the ability to switch between rows following and turning from the end of one row to beginning of the next. Inside a row, the vehicle sensed a structured row of trees even if some trees are missing. This helped the vehicle to locate itself and follow the desired trajectory. Outside the rows, the vehicle could not use the rows of trees as a guide due to incomplete information about its surroundings. While manoeuvring between rows (see Fig. 5), the vehicle could only use its steering encoder and odometer to position itself through the desired trajectory (in case a positioning system such as RTK-GPS is not in use).

When the high-level of consistent performance is the objective, manoeuvring between rows becomes critical especially when the region is slippery since it prevents the vehicle following the desired trajectory and reaching the target position (Fig. 5). The reasons, why the vehicle may not be able to reach to the target region, can be listed as follows:

- Sensors used for row detection may give illusory information about the next row; this causes the vehicle to enter the wrong row.

- The vehicle outside the desired path may perform a sudden and sharp turn in order to make its heading parallel to the row to be tracked; this sharp steering may damage the steering mechanism.

- The location of the target region is calculated according to the desired manoeuvring trajectory, which uses optimum arc characteristics (distance, radius, time, power, etc.); if the vehicle locates outside the desired manoeuvring trajectory or target region, it may spend more effort to do necessary corrections.

- Neglecting the slippage effects or poor slippage estimation may degrade the positioning and the dead-reckoning performances. 

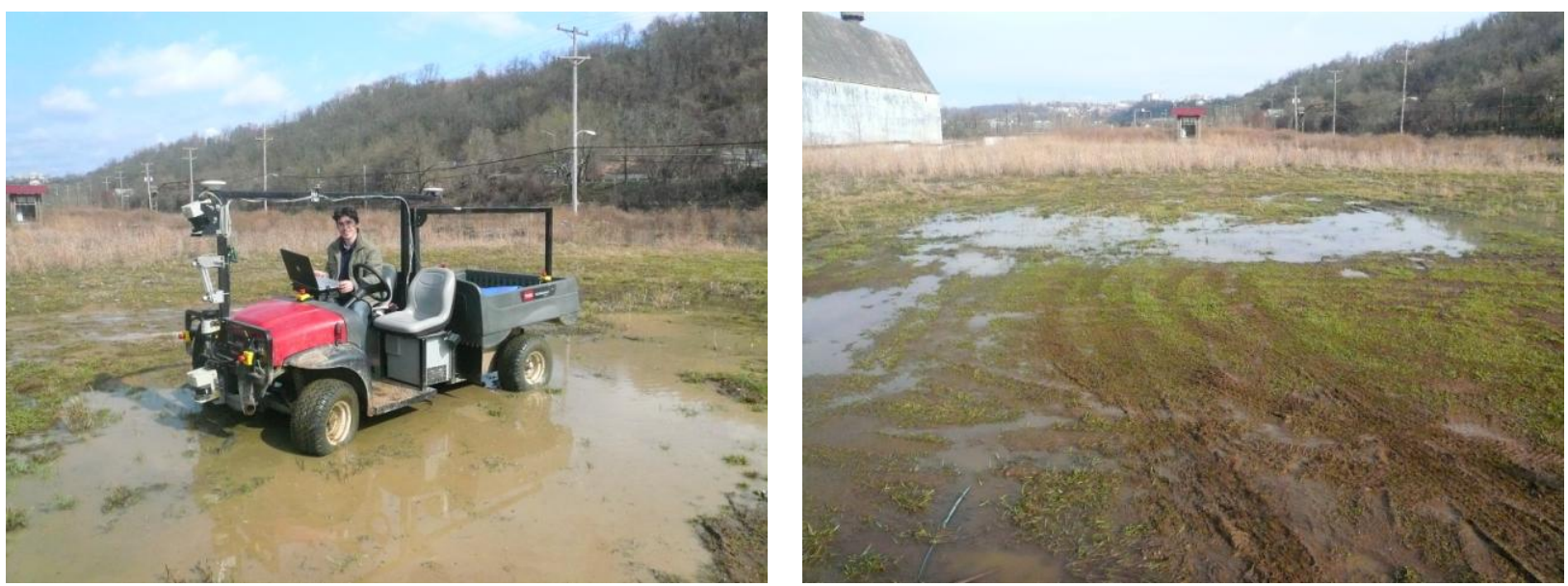

Fig, 16. Muddy surfaces in the experimental orchard area.

The experimental orchard area had muddy surface as shown in Fig. 16. Muddy surfaces cause two negative effects on vehicle motion; they cause it to slip and create sticky surface condition resulting in increased trajectory tracking error. As stated above, the slippage estimation procedure used the position information coming from RTK-GPS. Estimated values were fed into the system model and the required steering and heading velocity actions were generated.

The results of the orchard experiments are shown in Fig. 17. In order to highlight the slippage effects observed in trajectory tracking performance, the experiments were conducted twice. One experiment was performed using the system model which includes the estimated slippage information and one used the system model without the slippage estimation algorithm. Figure 17 shows that the target region (indicated by dash lined box) was reached when the slippage information was used. The vehicle did not track the desired path and locate itself in the target position when the slippage estimation algorithm was not integrated into the system model.

Slip velocities estimated in longitudinal (X) and lateral (Y) directions are shown in Figs. 18(a) and 18(b). Estimated sideslip angles at the rear of the vehicle are given in Fig. 18(c). To show the longitudinal and lateral slip velocities, the heading (forward) speed of the vehicle is given in Fig. 18(d).

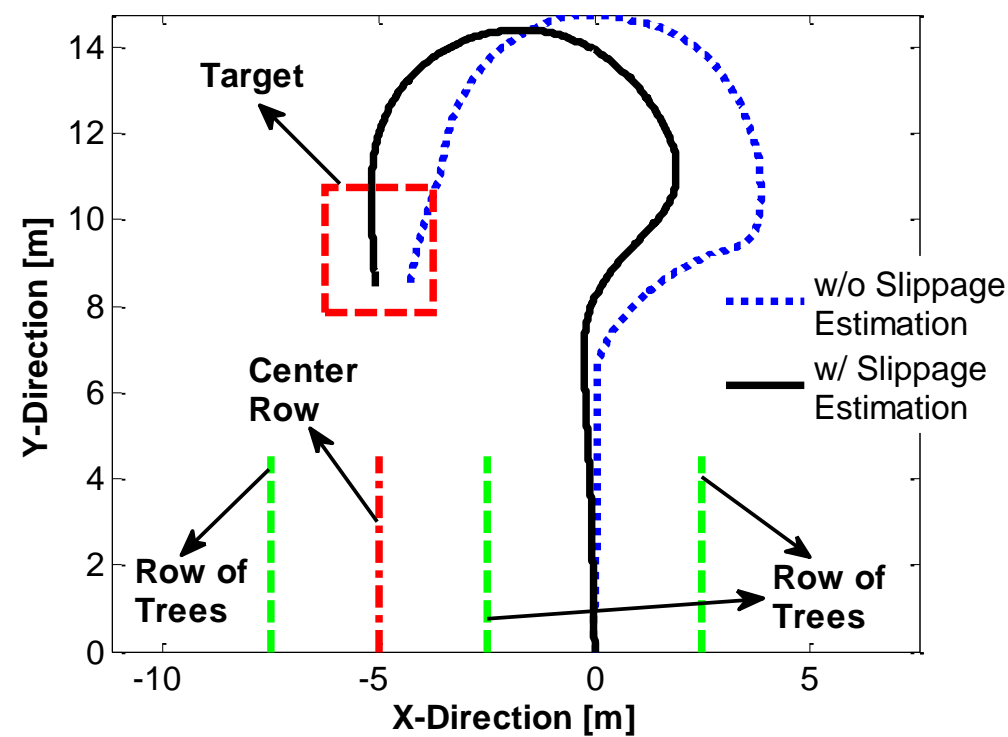

Fig. 17. Row following and turning in the experimental orchard area. 


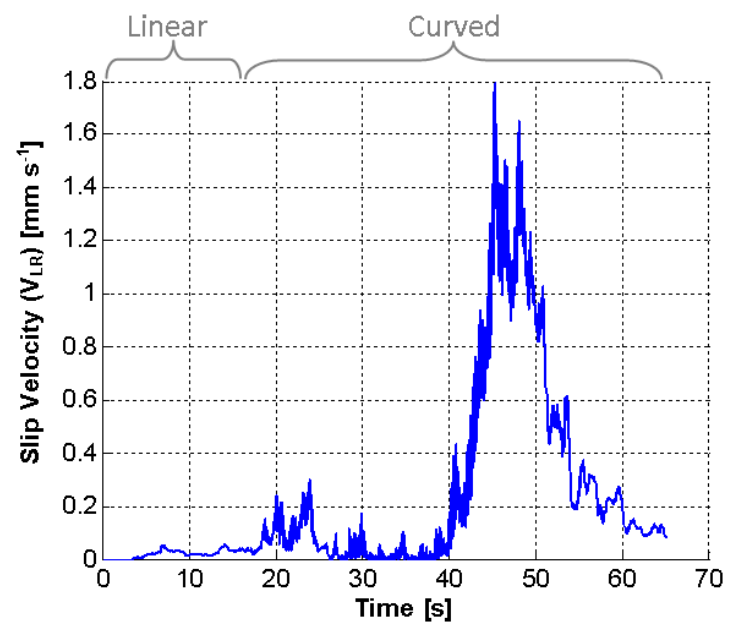

(a)

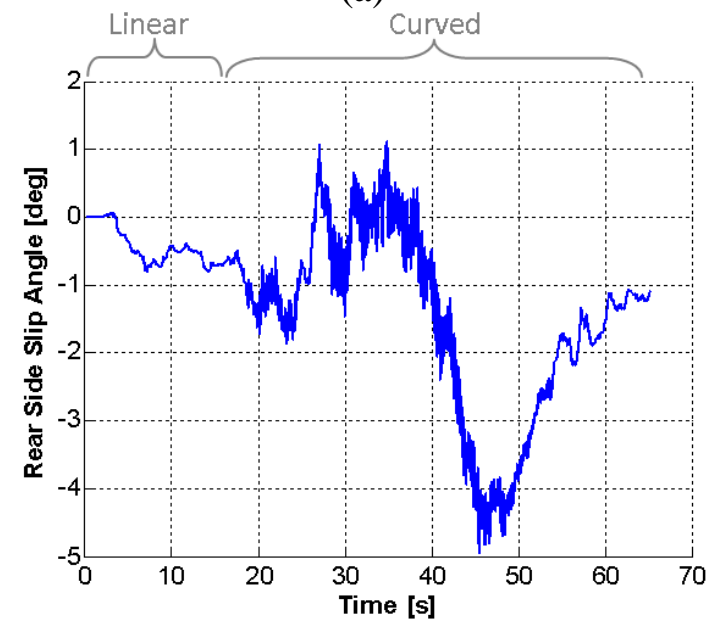

(c)

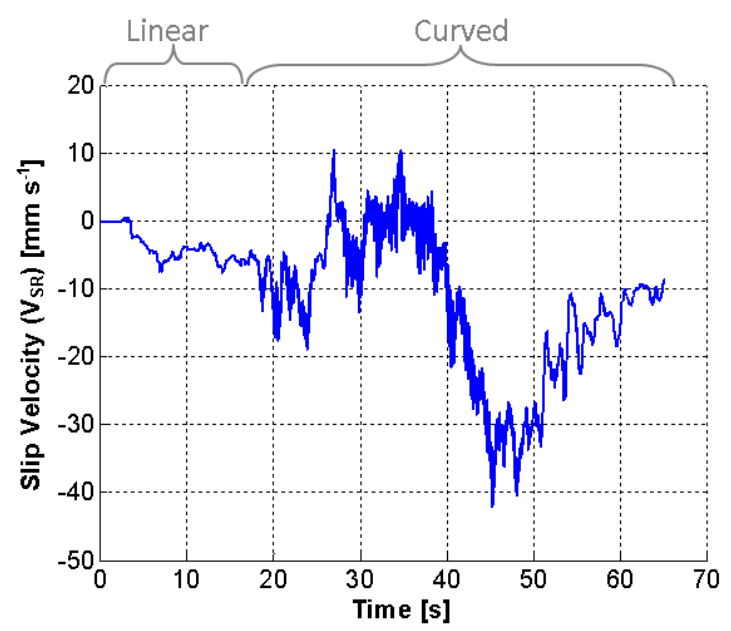

(b)

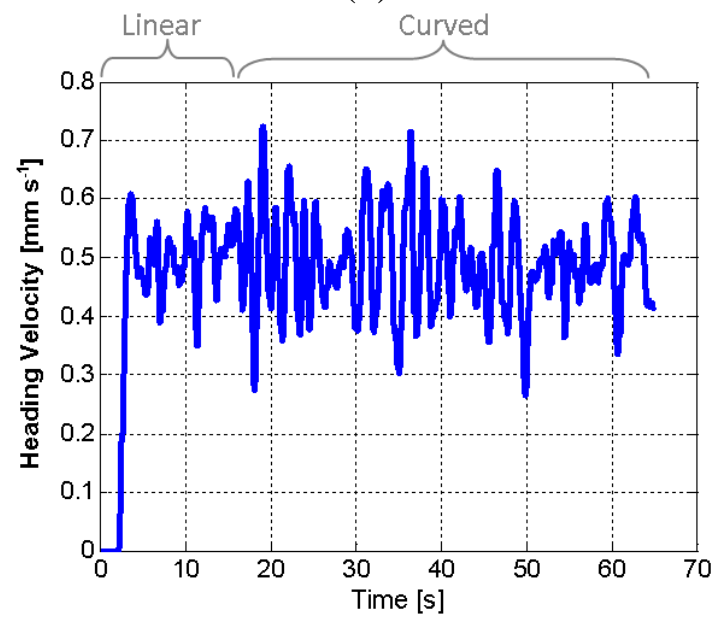

(d)

Fig. 18. (a) Estimated slip velocities in longitudinal direction, (b) estimated slip velocities in lateral direction, (c) estimated sideslip angle at the rear of the vehicle, (d) heading (i.e. forward) velocity of the vehicle.

The orchard experiments verified the intuitive fact that slippage prevents the vehicle tracking the desired trajectory accurately and reaching its target position. Taking slippage estimation into account in the system model enabled the controller to generate required control commands to follow the desired trajectory. It also gave the vehicle ability to reach the target position with the desired heading angle that is parallel to the row to be entered.

In order to demonstrate the repeatability and reproducibility of the system proposed, ten orchard experiments were conducted and the results are shown in Fig. 19. Five experiments were carried out using the system model which incorporated with the slippage estimation algorithm (red-coloured filled circles). The other five experiments were performed without incorporating the slippage information (blue-coloured filled circles). The objective of the experiments was to track a desired bulb-shaped trajectory and to access to the target box (as illustrated in Fig. 17). As stated above, the position of the target box is important since its centre coincides with the centre of row to be entered. For minimum steering action, the vehicle should place itself inside the target box with the minimum heading angle error. In Fig. 19, the end positions, heading angles of the vehicle and the position of neighbour row are shown. These results demonstrate that slippage prevents the vehicle following a reference trajectory and reaching the desired target box with the correct heading angle and integration of slippage information significantly improves row-changing behaviour. 
The open space was covered with snow and has inclined regions; whereas the experimental orchard field had a muddy surface. The open space preliminary experiments were conducted to adapt the proposed methods into the vehicle and to see if safe operation could be achieved or not. Comprehensive tests were carried out in an orchard environment. A row detection system based on a laser range finder was developed to detect tree trunks and create centre lines through the rows of trees. After following the centre line of a row and coming to its end, the vehicle had to leave the row and turned into the next. The orchard vehicle was equipped with a RTK-GPS system which served as the source of ground truth and was used as the position source for the slippage estimation procedure.

The results of experiments conducted in open space, and in the orchard showed that a significant amount of slippage occurred during turning which dramatically degraded the ability to track a predefined trajectory. They also indicated that if the slippage estimation was integrated into a system model, the vehicle could be controlled with less positioning errors. It is concluded that vehicle positioning accuracy, performance and repeatability for trajectory tracking in an orchard environment (i.e. on a muddy/snowy surface) can be improved by adopting the approach presented in this work. 


\section{Acknowledgments}

This work was partially supported by the US Department of Agriculture under grant no. 200851180-04876. The first author, Gokhan Bayar, would also like to thank to the Scientific and Technological Research Council of Turkey and Middle East Technical University for their financial supports.

\section{References}

Dubins, L.E. (1957). On curves of minimal length with a constraint on average curvature, and with prescribed initial and terminal positions and tangents, American Journal of Mathematics 79(3): 497516.

Kanayama, Y. and Hartman, B. I. (1989). Smooth local path planning for autonomous vehicles, IEEE International Conference on Robotics and Automation, Scottsdale, AZ, pp. 1265-1270.

Shin, D. H. and Singh, S. (1990). Path generation for robot vehicles using composite clothoid segments, Tech. Report CMU-RI-TR-90-31, Robotics Institute, Carnegie Mellon University.

Aleksander, H., Melinda, D. S. (2000). Estimation of vehicle side slip angle and yaw rate, SAE 2000 World Congress, Detroit, Michigan, pp. 1-7.

Ryu, J., Rossetter, E. J., Gerdes, J. C. (2002). Vehicle sideslip and roll parameter estimation using GPS, AVEC 6th Int. Symposium on Advanced Vehicle Control, Hiroshima, Japan.

Lindgren, D. R., Hague, T., Smith, P. J. P., Marchant, J. A. (2002). Relating torque and slip in an odometric model for an autonomous agricultural vehicle, Autonomous Robots, 13:73-86.

Joanny, S., Ali, C., Dominique, M. (2003). Vehicle sideslip angle observers, European Control Conference (ECC), Cambridge, UK, pp. 2993-2998.

Fang, H. (2004). Automatic guidance of farm vehicles in presence of sliding effects, A Scientific Report for the Post-Doc Research Project of Al Terrain Autonomous Vehicle Control.

Fang, H., Lenain, R., Thuilot, B., and Martinet, P. (2005). Robust adaptive control of automatic guidance of farm vehicles in the presence of sliding, IEEE International Conference on Robotics and Automation, Barcelona, Spain.

Lenain, R., Thuilot, B., Cariou, C., Martinet, P. (2006). Sideslip angles observer for vehicle guidance in sliding conditions: Application to agricultural path tracking tasks, IEEE International Conference on Robotics and Automation, Orlando, Florida, pp. 3183-3188.

Fang, H., Fan, R., Thuilot, B., Martinet, P. (2006). Trajectory tracking control of farm vehicles in presence of sliding, Robotics and Autonomous Systems, 54(10):828-839.

Barawid, O., Mizushima, A., Ishii, K., and Noguchi, N. (2007). Development of an autonomous navigation system using a two-dimensional laser scanner in an orchard application, Biosystems Engineering, 96(2):139-149.

Baffet, G., Charara, A., Lchner, D. (2008). Estimation of tire-road forces and vehicle sideslip angle, Advances in Robotics, Automation and Control, Book edited by: Jesus Aramburo and Antonio Ramirez Trevino, I-Tech, Vienna, Austria. 
Chen, B. C., Hsieh, F. C. (2008). Sideslip angle estimation using extended Kalman filter, Vehicle System Dynamics, 46:353-364.

Eaton, R., Katupitiya, J., Pota, H., Siew, K. W. (2009). Robust sliding mode control of an agricultural tractor under the influence of slip, IEEE/ASME International Conference on Advanced Intelligent Mechatronics, Singapore, pp. 1873-1878.

Grip, H. F., Imsland, L., Johansen, T. A., Kalkkuhl, J. C., Suissa, A. (2009). Vehicle sideslip estimation: Design, implementation and experimental validation, IEEE Control Systems Magazine, 29(5):36-52.

Iijima, T., Raksincharoensak, P., Michitsuji Y., Nagai, M. (2010). Vehicle sideslip angle estimation methodology using a drive recorder, Journal of Vibration and Control, 16(4):571-583.

Matveev, A. S., Hoy, M., Savkin, A. V. (2010). Mixed nonlinear sliding mode control of an unmanned farm tractor in the presence of sliding, 11th Int. Conf. Control, Automation, Robotics and Vision Singapore, pp. 927-932.

Lenain, R., Thuilot, B., Cariou, C., Martinet, P. (2010). Mixed kinematic and dynamic sideslip angle observer for accurate control of fast off-road mobile robots, Journal of Field Robotics, 27(2):181-196.

Singh, S., Bergerman, M., Cannons, J., Grocholsky, B., Hamner, B., Holguin, G., Hull, L., Jones, V., Kantor, G., Koselka, H., Li, G., Owen, J., Park, J., Shi, W., Teza, J. (2010). Comprehensive automation for specialty crops: Year 1 results and lessons learned, Journal of Intelligent Service Robotics, Special Issue on Agricultural Robotics, 3(4):245-262.

Hamner, B., Bergerman, M., and Singh, S. (2011). Autonomous orchard vehicles for specialty crops production, ASABE Annual International Meeting, Louisville, KY, USA.

Hamner, B., Bergerman, M., Singh, S. (2012). Specialty Crop Automation with Autonomous Vehicles, International Conference on Robotics and Automation, St. Paul, MN, pp. 1829-1835.

Davis, B. (2012). CMU-led automation program puts robots in the field, AUVSI's Unmanned Systems: Mission Critical, 2(1):38-40.

Freitas, G., Hamner, B., Bergerman, M., Singh, S. (2012). A practical obstacle detection system for autonomous orchard vehicles, IEEE/RSJ International Conference on Intelligent Robots and Systems, Algarve, Portugal.

Kanghyun, N., Sehoon, O., Hiroshi, F., Yoichi, H. (2012). Estimation of sideslip and roll angles of electric vehicles using lateral tire force sensors through rls and kalman filter approaches, IEEE Transactions on Industrial Electronics, 60(3): 988-1000.

Bayar, G. (2013). Long distance autonomous trajectory tracking for an orchard vehicle, Industrial Robot: An International Journal, 40(1):27-40.

Bayar, G., Bergerman, M., Koku, A. B., Konukseven, E. i. (2015). Localization and control of an autonomous orchard vehicle, Computers and Electronics in Agriculture, 115:118-128.

ROS (2015). Robot Operating System. Available from www.ros.org. Accessed: 24.12.15. 